\title{
Transactions
}

Cite this: Dalton Trans., 2012, 41, 3515

www.rsc.org/dalton

PAPER

\section{MOP-phosphonites: A novel ligand class for asymmetric catalysis $\uparrow$}

\author{
Arne Ficks, ${ }^{a}$ Rachel M. Hiney, ${ }^{b}$ Ross W. Harrington, ${ }^{a}$ Declan G. Gilheany ${ }^{b}$ and Lee J. Higham ${ }^{* a}$ \\ Received 18th November 2011, Accepted 21st December 2011 \\ DOI: $10.1039 / \mathrm{c} 2 \mathrm{dt} 12214 \mathrm{f}$
}

Chiral phosphonite ligands $\left(S, R_{\mathrm{b}}\right)-\mathbf{5 a},\left(S, S_{\mathrm{b}}\right)-\mathbf{5} \mathbf{b},\left(R, R_{\mathrm{b}}\right)-\mathbf{6 a}$ and $\left(R, S_{\mathrm{b}}\right)-\mathbf{6} \mathbf{b}$ are introduced, comprising a MOP-type backbone with a binol-based binaphthyl group bound to the phosphorus. Their reaction with $\left[\operatorname{Pd}\left(\eta^{3}-\mathrm{C}_{4} \mathrm{H}_{7}\right) \mathrm{Cl}\right]_{2}$ affords $\eta^{3}$-methallylpalladium chloride complexes $\mathbf{7} \mathbf{a} / \mathbf{b}$ and $\mathbf{8 a} / \mathbf{b}$ which have been isolated and structurally characterised. Solid-state and solution studies indicate subtle differences in their coordination behaviour, which ultimately affects their efficacy in the asymmetric hydrosilylation of styrene.

\section{Introduction}

Chiral monophosphorus ligands have attracted considerable attention as their transition metal complexes have been found to be valuable catalysts in a variety of organic transformations. ${ }^{1}$ Binaphthyl-derived phosphorus ligands based on phosphine, ${ }^{2}$

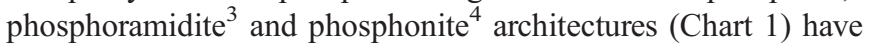
all demonstrated their effectiveness in asymmetric catalysis.

Primary phosphines have a reputation for being difficult compounds to work with owing to their perceived high reactivity towards oxygen. ${ }^{5}$ Hence, they are somewhat under-represented as synthons in synthetic chemistry, despite the two $\mathrm{P}-\mathrm{H}$ bonds being easily functionalised. A few examples of user-friendly, airstable primary phosphines have however been reported, ${ }^{6}$ and we were the first to describe enantiopure analogues; ${ }^{7}$ we subsequently published a DFT-based model to help rationalise this stability. ${ }^{8}$ We also recently described how air-stable, chiral primary phosphines $(S)-\mathbf{1}$ and $(R)-\mathbf{2}$ could form novel

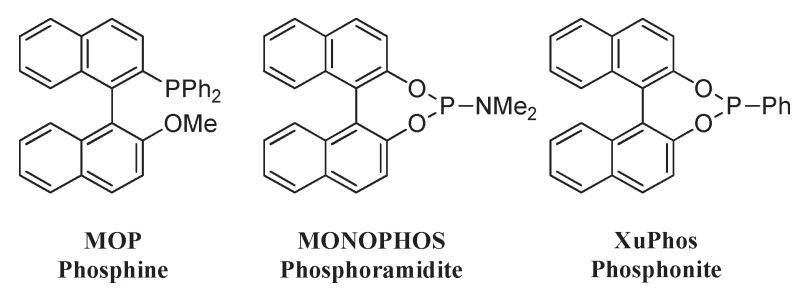

Chart 1 Established classes of binaphthyl monophosphorus ligands.

\footnotetext{
${ }^{a}$ School of Chemistry, Bedson Building, Newcastle University, Newcastle Upon Tyne, NE17RU, UK.E-mail:lee.higham@ncl.ac.uk; Tel: (+44) 1912225542

${ }^{b}$ School of Chemistry and Chemical Biology, The Centre for Synthesis and Chemical Biology, University College Dublin, Belfield, Dublin 4, Ireland

$\dagger$ Electronic supplementary information (ESI) available: Full experimental and crystallographic data. CCDC reference numbers 840097 (7a), 840098 (7b), 854373 (8a) and 840100 (8b). For ESI and crystallographic data in CIF or other electronic format see DOI: 10.1039/ c2dt12214f
}

phosphiranes with unusually high oxidative and thermal stability. ${ }^{9}$ We were therefore keen to establish whether we could transform $(S)$-1 and $(R)-2$ into their dichlorophosphines, and ultimately access MOP/XuPhos-type hybrids (Chart 1), or whether their resistance to oxidation would render them unreactive to halogen sources. This work focuses on the synthesis and characterisation of a novel MOP-phosphonite ligand class. The coordination chemistry of their methallylpalladium complexes is described and we have therefore investigated their potential as asymmetric ligands in the catalytic hydrosilylation of styrene.

\section{Results and discussion}

Four MOP-phosphonite ligand derivatives have each been synthesised in a straightforward two-step, one-pot reaction approach. The ligands differ in the substituent on the 2 '-position of the MOP-backbone ( $\mathrm{H}$ or $\mathrm{OMe})$ and in the stereochemistry of the affiliated hydroxyl compound $((R)$ - or $(S)$-binol). By comparing the two pairs of diastereomeric compounds with each other, we were able to elucidate the effect of the second stereocentre.

Following the synthetic method of Weferling, ${ }^{10,11}(S)-\mathbf{1}$ and $(R)-2$ were treated with phosphorus pentachloride to give the dichlorophosphines $(S)$-3 and $(R)-\mathbf{4}$ in quantitative conversion. Subsequent addition of $(R)$-binol under basic conditions afforded the MOP-phosphonite hybrids $\left(S, R_{\mathrm{b}}\right)-\mathbf{5 a}$ and $\left(R, R_{\mathrm{b}}\right)-\mathbf{6 a}$ as white solids (Scheme 1). Their diastereomers $\left(S, S_{\mathrm{b}}\right)-\mathbf{5} \mathbf{b}$ and $\left(R, S_{\mathrm{b}}\right)-\mathbf{6 b}$ were afforded from reactions with $(S)$-binol, again in high yield.

The ${ }^{31} \mathrm{P}$ NMR spectroscopic data for the phosphonites are as expected, showing characteristic resonances ( $\delta=177.4 \mathrm{ppm}$ for $\left(S, R_{\mathrm{b}}\right)-\mathbf{5 a} ; 177.8 \mathrm{ppm}$ for $\left(R, R_{\mathrm{b}}\right)-\mathbf{6 a} ; 175.7 \mathrm{ppm}$ for $\left(S, S_{\mathrm{b}}\right)-\mathbf{5} \mathbf{b}$; 177.9 for $\left.\left(R, S_{\mathrm{b}}\right)-\mathbf{6 b}\right)$. Notably, all the ligands could conveniently be handled in air; in common reagent grade solvents no hydrolysis products were found after 24 h. ${ }^{12}$ We next studied the coordination chemistry of the phosphonites; reaction of two equivalents of the pertinent ligand with $\left[\mathrm{Pd}\left(\eta^{3}-\mathrm{C}_{4} \mathrm{H}_{7}\right) \mathrm{Cl}\right]_{2}$ resulted in the rapid, quantitative formation of $7 \mathbf{a}, 7 \mathbf{b}, 8 \mathbf{a}$ and $8 \mathbf{b}$ (Scheme 2) as shown by NMR, HRMS and X-ray crystallography. 


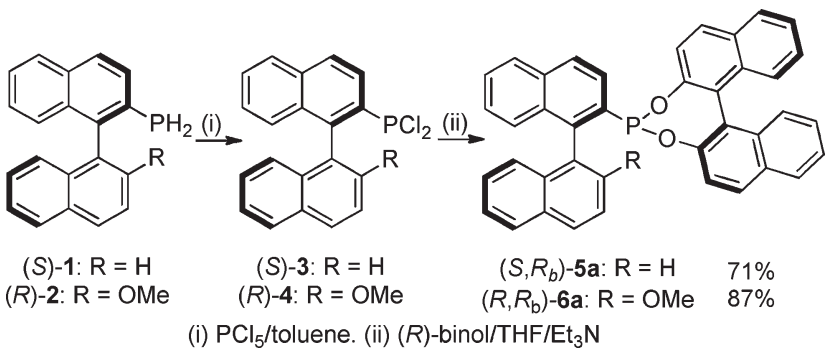

Scheme 1 The synthesis of phosphonites $\left(S, R_{\mathrm{b}}\right)-\mathbf{5 a}$ and $\left(R, R_{\mathrm{b}}\right)-\mathbf{6 a}$.

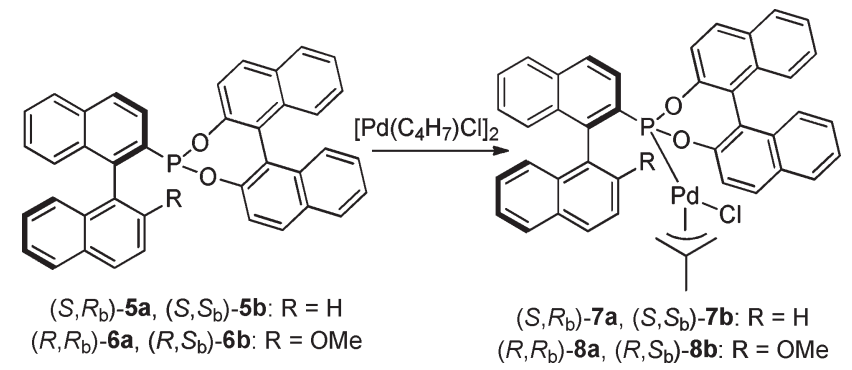

Scheme 2 The synthesis of the complexes $7 \mathbf{a}, 7 \mathbf{b}, \mathbf{8 a}$ and $\mathbf{8 b}$.

In all four complexes the X-ray crystal structure reveals the methyl group on the allyl fragment points towards the binol component of the ligand (see Fig. 1-4). The torsion angles of the two binaphthyl fragments present are significantly different from each other. The unstrained MOP portion preserves an almost right angle while the torsion of the binol moiety is enforced by the bonding of both oxygen atoms to the phosphorus, and thus appears acutely angled. The ligands coordinate via the phosphorus donor atom in an expected monodentate manner to form a pseudo-square-planar configuration around the palladium. $\mathrm{Pd}-$ $\mathrm{P}$ bond lengths are similar in all complexes $(2.2354(7)$ to 2.2542 (8) $\AA$ ) and are found to be shorter than for the two MOP-phosphine allylpalladium complexes previously reported (2.3098(9)

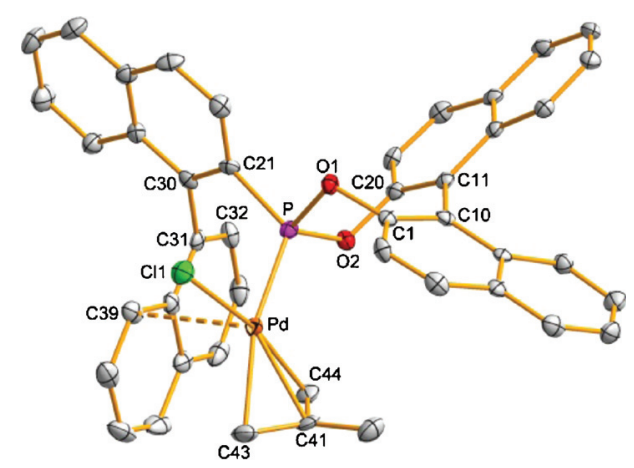

Fig. 1 Molecular structure of 7 a with $50 \%$ probability displacement ellipsoids. Hydrogen atoms have been omitted for clarity. Selected bond distances $(\AA)$ and angles (deg): $\mathrm{Pd}-\mathrm{P} 2.2476(7), \mathrm{Pd}-\mathrm{Cl}(1)$ 2.3854(7), Pd-C(44) 2.093(2), Pd-C(43) 2.206(2), C(41)-C(43) 1.384(4), C(41)-C (44) 1.415(4), Pd‥C(39) 3.0653(1); P-Pd-Cl(1) 93.67(2), P-Pd-C(44) 98.23(9), $\mathrm{Cl}(1)-\mathrm{Pd}-\mathrm{C}(43)$ 101.60(7), C(43)-Pd-C(44) 66.68(11), C (21) $-\mathrm{C}(30)-\mathrm{C}(31)-\mathrm{C}(32)-101.2(3), \quad \mathrm{C}(1)-\mathrm{C}(10)-\mathrm{C}(11)-\mathrm{C}(20)-51.2$ (3). and 2.328(1) $\AA$ ); ${ }^{13}$ however, shortened Pd-P bond lengths are anticipated for phosphonite complexes due to their stronger $\pi$-acceptor character. ${ }^{14} \mathrm{Pd}-\mathrm{C}$ bond lengths show the dominant trans effect of the P-ligand relative to the chloride; the bonds trans to the phosphorus are about $0.1 \AA$ longer compared to the bonds in the cis position. In all complexes the position of the palladium centre is face-to-face with the lower naphthyl moiety of the MOP fragment of the ligand. However, the exact position of the methallylpalladium moiety is somewhat diverse. When the phosphonite consists of a $(R)$-binol fragment (7a/8a), the palladium is situated towards the back of the lower napthyl ring (Fig. 1 and Fig. 3); in $\mathbf{7 b} / \mathbf{8 b}$, as a consequence of the opposite binol stereochemistry, the metal is instead located above the front of this group (Fig. 2 and Fig. 4). The distance of the palladium atom from the naphthyl group ranges from $3.0653(1) \AA$ in $\mathbf{7 a}$ to $3.4458(1)$ in $\mathbf{8 b}$.

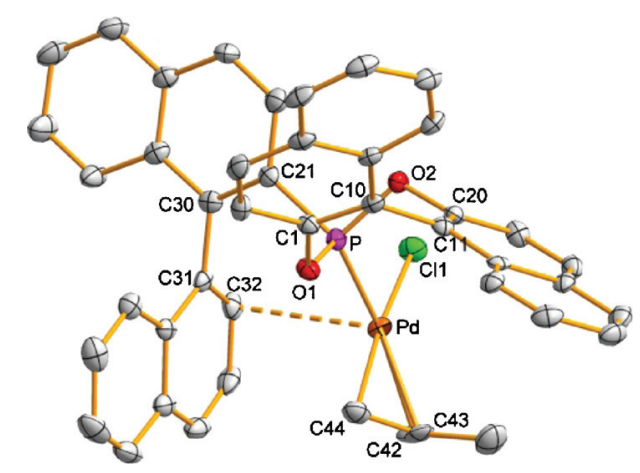

Fig. 2 View of the molecular structure of $7 \mathbf{b}$. Hydrogen atoms have been omitted for clarity. Selected bond distances $(\AA)$ and angles $(\mathrm{deg})$ : Pd-P 2.2354(7), Pd-Cl(1) 2.3730(8), Pd-C(44) 2.078(3), Pd-C(42) 2.198(3), C(42)-C(43) 1.387(4), C(43)-C(44) 1.409(4), $\mathrm{Pd} \cdots \mathrm{C}(32)$ 3.1412(1); P-Pd-Cl(1) 94.51(3), P-Pd-C(44) 96.49(9), Cl(1)-Pd-C(42) 101.71(9), C(42)-Pd-C(44) 67.31(13), C(21)-C(30)-C(31)-C(32) -90.5(4), C(1)-C(10)-C(11)-C(20) 52.1(4).

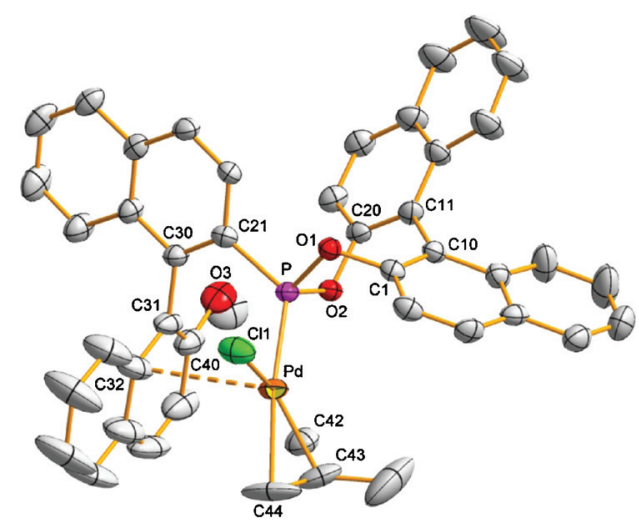

Fig. 3 View of the molecular structure of $\mathbf{8 a}$. Hydrogen atoms and cocrystallised solvent have been omitted for clarity. Selected bond distances $(\AA)$ and angles (deg): Pd-P 2.2363(8), Pd-Cl(1) 2.3755(10), PdC(42) 2.104(4), Pd-C(44) 2.185(4), C(42)-C(43) 1.415(6), C(43)-C(44) 1.381(7), $\mathrm{Pd} \cdots \mathrm{C}(32)$ 3.4018(1); P-Pd-Cl(1) 93.29(3), P-Pd-C(42) 98.24(12), Cl-Pd-C(44) 101.41(16), C(42)-Pd-C(44) 67.08(19), C (21)-C(30)-C(31)-C(40) -98.0(4), C(1)-C(10)-C(11)-C(20) -52.1(4). 


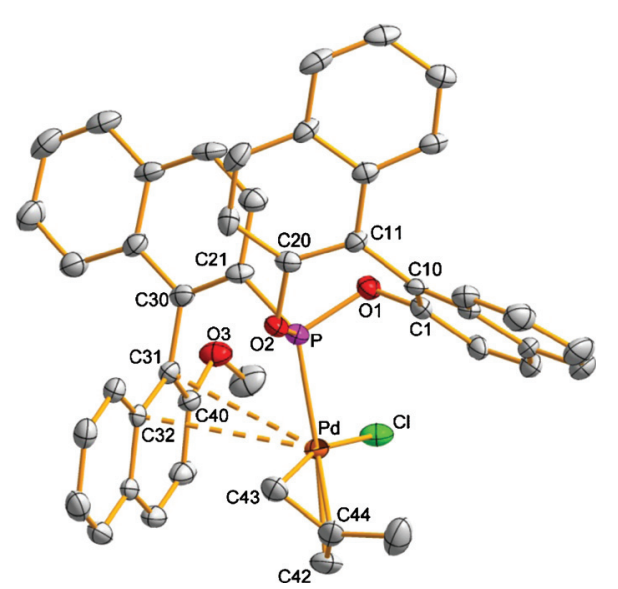

Fig. 4 View of the molecular structure of $\mathbf{8 b}$. Hydrogen atoms and cocrystallised solvent have been omitted for clarity. Selected bond distances $(\AA)$ and angles (deg): Pd-P 2.2542(8), Pd-Cl 2.3675(7), Pd-C (43) 2.101(3), Pd-C(42) 2.177(3), C(42)-C(44) 1.402(5), C(43)-C(44) 1.412(4), $\mathrm{Pd} \cdots \mathrm{C}(31)$ 3.4458(1), $\mathrm{Pd} \cdots \mathrm{C}(32)$ 3.4793(0); $\mathrm{P}-\mathrm{Pd}-\mathrm{Cl} 98.46$ (3), P-Pd-C(43) 96.80(8), Cl-Pd-C(42) 97.23(9), C(42)-Pd-C(43) 67.39(12), C(21)-C(30)-C(31)-C(40) -98.9(3), C(1)-C(10)-C(11)-C (20) $53.8(4)$.

The NMR spectra of complexes 7a and 8a show the presence of two isomers in a 97/3 and 93/7 ratio, respectively, which can be rationalised by a rotation of the allyl moiety. NOE contacts between each pair of syn and anti protons and between the syn allyl protons and the central methyl group, in addition to allyl proton-phosphorus coupling, allowed for a complete NMR assignment of the allyl signals (Table S2 $\dagger$ ). The protons trans to the phosphorus ligand are deshielded and show coupling with ${ }^{31} \mathrm{P}$ whereas the cis protons are observed as singlets at higher field. ${ }^{15}$ The ${ }^{13} \mathrm{C}$ chemical shifts for the atoms in terminal allyl positions are consistent with those reported for similar complexes: ${ }^{16} 77-79 \mathrm{ppm}\left({ }^{2} J_{\mathrm{CP}} \approx 46 \mathrm{~Hz}\right)$ for the allyl carbons trans to the P-donor, and 56-59 ppm $\left({ }^{2} J_{\mathrm{CP}} \approx 5 \mathrm{~Hz}\right)$ for the cis allyl carbons. The phase-sensitive NOESY spectrum of 8a shows exchange peaks due to interconversion of the two isomers (see Fig. $\mathrm{S} 2 \dagger)$. Quantitative analysis of the peak integrals yielded exchange rate constant values of $k_{\mathrm{AB}} \approx 0.12 \mathrm{~s}^{-1}$ and $k_{\mathrm{BA}} \approx$ $1.5 \mathrm{~s}^{-1}$ at room temperature.

The selective syn/anti exchange of the allyl protons cis to the phosphorus is caused by the well-known $\eta^{3}-\eta^{1}-\eta^{3}$ mechanism (Scheme 3); the protons in the trans position exchange syn/syn and anti/anti, while the cis protons exchange syn/anti due to the selective opening of the allyl ligand. ${ }^{16,17}$ The selectivity of the process is due to the stronger trans effect of the P-donor ligand compared to the chloro ligand. An apparent allyl rotation mechanism which is often observed in N-donor ligands requires a syn/ syn and anti/anti exchange for both cis and trans allyl protons
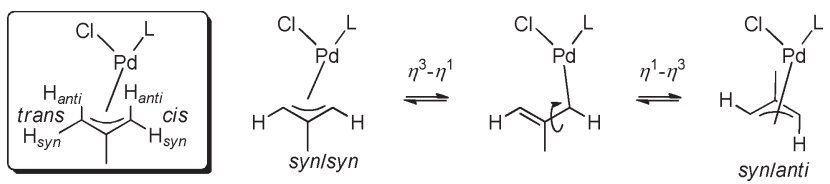

Scheme 3 Syn/anti exchange mechanism in allyl palladium complexes.
Table 1 Selected ${ }^{13} \mathrm{C}$ NMR data $(\delta$ in ppm) for ligands $\mathbf{6 a}$ and $\mathbf{6 b}$ and their palladium complexes $8 \mathbf{8 a}, \mathbf{8 b}, \mathbf{9 a}$ and $\mathbf{9 b}\left(\mathrm{CD}_{2} \mathrm{Cl}_{2}, 21^{\circ} \mathrm{C}\right)$

\begin{tabular}{lllllll}
\hline Carbon & $\mathbf{6 a}$ & $\mathbf{8 a}^{a}$ & $\mathbf{9} \mathbf{a}^{b}$ & $\mathbf{6 b}$ & $\mathbf{8 b}$ & $\mathbf{9 b}^{b}$ \\
\hline $1^{\prime}$ & 118.9 & 118.8 & $104.6 /$ n.d. $^{c}$ & 119.0 & 118.2 & $103.4 / 104.5$ \\
$2^{\prime}$ & 156.6 & 156.4 & $157.2 / 159.0$ & 154.9 & 156.2 & $156.6 / 154.8$ \\
$3^{\prime}$ & 112.8 & 113.1 & $114.9 / 114.5$ & 113.0 & 113.5 & $115.3 / 115.9$ \\
$4^{\prime}$ & 130.8 & 131.0 & $134.6 / 134.6$ & 130.7 & 129.7 & $134.9 / 134.3$ \\
$9^{\prime}$ & 134.5 & 134.2 & $131.5 / 131.4$ & 135.0 & 135.5 & $132.7 / 133.7$ \\
$10^{\prime}$ & 128.2 & 127.0 & $128.4 / 128.4$ & 128.3 & 128.2 & $129.3 / 130.0$
\end{tabular}

${ }^{a}$ Major isomer. ${ }^{b}$ Two isomers were observed due to allyl rotation. ${ }^{c}$ No distinctive assignment due to signal overlap and peak broadening.

and can be ruled out here. ${ }^{18}$ Note that conversely for complexes $\mathbf{7 b}$ and $\mathbf{8 b}$, we observe only a single isomer in each case.

MOP ligands can display unexpected bonding characteristics upon metal complexation, using their aromatic backbone to coordinate to a vacant metal site, as shown by several groups who observed binding in a chelating $\mathrm{P}, \mathrm{C}-\sigma$-donor or $\mathrm{P}, \mathrm{C}-\pi$-olefin bidentate fashion. ${ }^{13 \mathrm{~b}, 16,19,20}$ When the chloride counterions of complexes 8a and $\mathbf{8 b}$ were exchanged for the non-coordinating BArF anion to give complexes $\mathbf{9 a}$ and $\mathbf{9 b}$, the $\mathrm{C}^{\prime}$ carbon shifted by -14.3 (9a) or $-15.6 /-14.5 \mathrm{ppm}$ (for the two isomers of $9 \mathbf{b}$ ) to lower frequency compared to the free ligand, indicative of greater $\mathrm{sp}^{3}$ character. The chemical shift of the $\mathrm{C} 2$ ' carbon remains almost unchanged (Table 1).

In agreement with studies made by Pregosin and co-work$\mathrm{ers}^{20 \mathrm{~b}}$ we therefore propose a weak $\eta^{1}$ binding mode of the lower naphthyl ring via the $\mathrm{C}-1^{\prime}$ carbon (Fig. 5, left). The downfield shifts of $3.8 \mathrm{ppm}$ in $\mathbf{9 a}$ and $4.2 / 3.6 \mathrm{ppm}$ in $\mathbf{9 b}$ for the C-4' carbon also suggests it carries some of the associated positive charge of the cation, again matching the aforementioned work. A section of the ${ }^{13} \mathrm{C}-{ }^{1} \mathrm{H}$ HMBC spectrum of $9 \mathbf{b}$ showing the indirect resonances of the $\mathrm{C} 1^{\prime}$ and $\mathrm{C} 2{ }^{\prime}$ carbons is given (Fig. 5, right).

The catalytic activity of the newly prepared ligands was tested in the asymmetric hydrosilylation of styrene. ${ }^{21}$ The catalysts were generated in situ from allylpalladium chloride dimer and the appropriate phosphonite. We chose a ligand to palladium ratio of $1: 1$ to form the catalyst precursors as the methallylpalladium complexes $\mathbf{7 a} / \mathbf{b}$ and $\mathbf{8 a} / \mathbf{b}$ had been obtained in this way (vide supra). We found that the activity of the catalyst was predominantly dependent on the substituent in the $2^{\prime}$-position of the
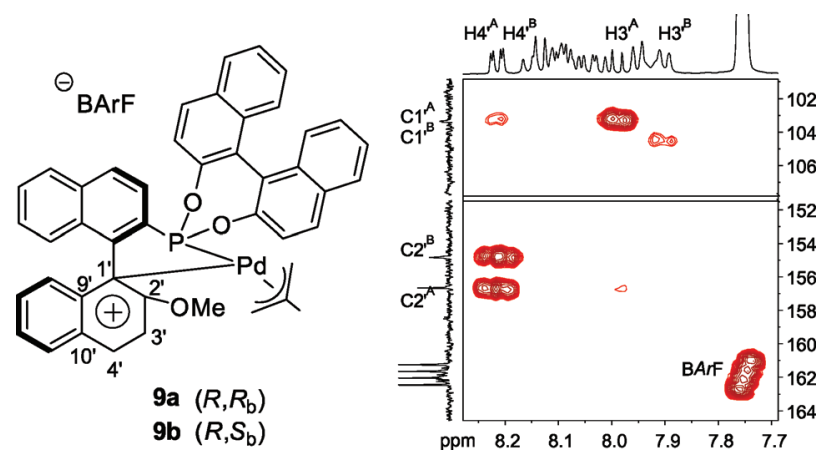

Fig. 5 Left: Proposed binding mode in $\mathbf{9 a} / \mathbf{b}$ using ${ }^{13} \mathrm{C}$ NMR data. Right: Section of the ${ }^{13} \mathrm{C}-{ }^{1} \mathrm{H}$ HMBC spectrum of $\mathbf{9 b}$ with key $\mathrm{C}^{\prime} / \mathrm{H}^{\prime}{ }^{\prime}$ and $\mathrm{C}^{\prime} / \mathrm{H} 4^{\prime}$ data shown; two isomers of $\mathbf{9 b}$ (labelled A, B) are present. 
Table 2 The palladium-catalyzed asymmetric hydrosilylation of styrene

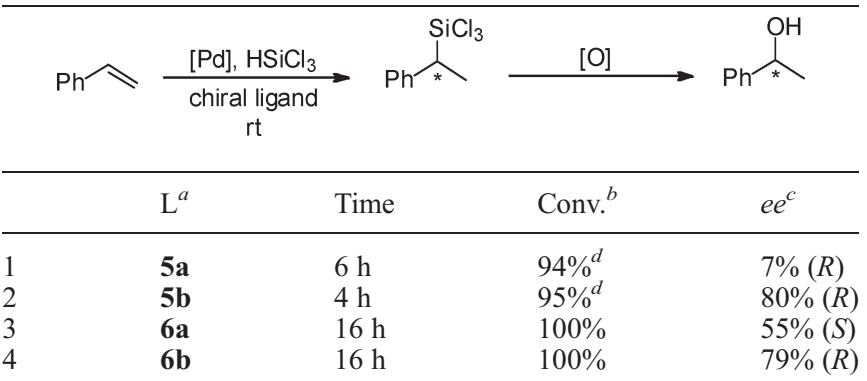

${ }^{a}$ The catalyst was generated in situ from the ligand $(0.25 \mathrm{~mol} \%)$ and $[\mathrm{Pd}$ $($ allyl $) \mathrm{Cl}]_{2}(0.125 \mathrm{~mol} \%)$, and reacted with styrene $(10.0 \mathrm{mmol})$ and trichlorosilane $(12.0 \mathrm{mmol}) .{ }^{b}$ Determined by ${ }^{1} \mathrm{H}$ NMR spectroscopy. ${ }^{c}$ Determined by chiral HPLC; absolute configuration assigned by comparing the retention times to literature data. ${ }^{23}{ }^{d}$ Complete conversion after additional $2 \mathrm{~h}$ reaction time.

ligand: H-MOP derivatives $\mathbf{5 a / b}$ showed a higher activity ( $>94 \%$ conversion after $6 \mathrm{~h}$ ) than their corresponding OMe-MOP derivatives $\mathbf{6 a} / \mathbf{b}$ (completed after $16 \mathrm{~h}$, Table 2 ). In contrast, the selectivity was mostly determined by the configuration of the adherent binol group. Enantioselectivities of 80 and $79 \%$ were achieved with the $(S)$-binol derivatives $\mathbf{5 b}$ and $\mathbf{6 b}$, while $(R)$ binol compounds $5 \mathbf{a}$ and $\mathbf{6 a}$ gave lower ee values. In previous studies H-MOP ligands have been found to be more selective in this transformation than their OMe-MOP counterparts, ${ }^{2 \mathrm{a}}$ hence our results for 5a (H-MOP derivative, low ee) and $\mathbf{6 b}$ (OMeMOP derivative, high ee) were somewhat surprising. Solid-state analysis of an H-MOP allylpalladium phosphine complex, ${ }^{13 \mathrm{~b}}$ found to be very selective in the same catalytic reaction, ${ }^{22}$ shows a similar $\mathrm{P} / \mathrm{Pd}$ environment to that found in the H-MOP complex 7b. In contrast, the palladium atom in $7 \mathbf{a}$ is located more towards the back of the lower naphthyl ring. Thus the subtle differences in the position of the palladium atom relative to the MOP fragment in the catalytically active species could be crucial in determining the reaction enantioselectivity, again emphasising that we are comparing pairs of diastereomers in this study; an examination of the wider implications of these results is now underway.

\section{Conclusions}

In summary, we have demonstrated that the air-stable primary phosphines $(S)$-1 and $(R)$-2 readily form their dichlorophosphine counterparts, which react as electrophiles with enantiopure binol to give novel chiral phosphonites. We have characterised each ligand as its methallylpalladium complex by NMR and X-ray crystallography, which together with detailed NMR experiments on related cationic complexes indicate the subtly different metal environment in each case and the P,C ligation of $\mathbf{9 a} / \mathbf{b}$. In the hydrosilylation of styrene, enantioselectivities of $80 \%$ were achieved in reactions that have not yet been optimised.

\section{Experimental}

\section{General}

All air- and/or water-sensitive reactions were performed under an $\mathrm{N}_{2}$ atmosphere using standard Schlenk line techniques. THF and
$\mathrm{CH}_{2} \mathrm{Cl}_{2}$ were dried over $\mathrm{Na} /$ benzophenone and $\mathrm{CaH}_{2}$ respectively, and distilled prior to use. Toluene (Acros) was purchased in an anhydrous state and stored over molecular sieves. The preparations of $(S)-\mathbf{1}$ and $(R)-\mathbf{2}$ were carried out using literature procedures. $^{7}$ All other chemicals were used as received without further purification. Flash chromatography was performed on silica gel from Fluorochem (silica gel, 40-63u, 60A, LC301). Thin-layer-chromatography was performed on Merck aluminium-based plates with silica gel and fluorescent indicator $254 \mathrm{~nm}$. For indicating, UV light or $\mathrm{KMnO}_{4}$ solution $(1.0 \mathrm{~g}$ $\mathrm{KMnO}_{4}, 6.7 \mathrm{~g} \mathrm{~K}_{2} \mathrm{CO}_{3}, 0.1 \mathrm{~g} \mathrm{NaOH}, 100 \mathrm{~mL} \mathrm{H}_{2} \mathrm{O}$ ) was used. Melting points were determined in open glass capillary tubes on a Stuart SMP3 melting point apparatus. Optical rotation values were determined on an Optical Activity Polaar 2001 device. ${ }^{1} \mathrm{H}$ NMR, ${ }^{11} \mathrm{~B}\left\{{ }^{1} \mathrm{H}\right\}$ NMR, ${ }^{13} \mathrm{C}\left\{{ }^{1} \mathrm{H}\right\}$ NMR, ${ }^{19} \mathrm{~F}$ NMR, and ${ }^{31} \mathrm{P}\left\{{ }^{1} \mathrm{H}\right\}$ NMR spectra were recorded on a JEOL Lambda $500\left({ }^{1} \mathrm{H}\right.$ $500.16 \mathrm{MHz})$ or JEOL ECS-400 $\left({ }^{1} \mathrm{H} 399.78 \mathrm{MHz}\right)$ spectrometer at room temperature $\left(2{ }^{\circ} \mathrm{C}\right)$ if not otherwise stated, using the indicated solvent as internal reference. Two-dimensional NMR experiments (COSY, NOESY, HSQC, HMBC) were used for the assignment of proton and carbon resonances, the numbering scheme is given in Fig. S1 $\uparrow$. Full range NOESY spectra were acquired with $512 \times 1024$ data points and a spectral width of 9.0 ppm; mixing times were chosen between 10 and $500 \mathrm{~ms}$. For the measurement of exchange rate constants in $\mathbf{8 a}$, the proton resonances of the methoxy group were used. Peak volumes were determined manually from the NOESY spectrum using MestReNova 6, and the rate constants were calculated with an estimated error of $10 \%$ using EXSYCalc. ${ }^{24}$ Mass spectrometry was carried out by the EPSRC National Mass Spectrometry Service Centre, Swansea. Analytical high performance liquid chromatography (HPLC) was performed on a Varian Pro Star HPLC equipped with a variable wavelength detector.

\section{Synthesis of $\left(S, R_{\mathrm{b}}\right)$-[1,1'-binaphthalene]-2,2'-diyl [1,1'-binaphthalen]-2-ylphosphonite (5a)}

$\mathrm{PCl}_{5}$ (458 mg, $2.20 \mathrm{mmol}$ ) was dissolved in toluene ( $\left.8 \mathrm{~mL}\right)$. (S)$1(286 \mathrm{mg}, 1.00 \mathrm{mmol})$ was added and the reaction mixture was left to stir for $45 \mathrm{~min}$. The volatiles were removed in vacuo to give $(S)-3\left({ }^{31} \mathrm{P}\left\{{ }^{1} \mathrm{H}\right\} \mathrm{NMR}, \mathrm{CDCl}_{3}: \delta=157.1 \mathrm{ppm}\right)$ as a yellow oil. THF ( $8 \mathrm{~mL}), \mathrm{NEt}_{3}(448 \mathrm{mg}, 0.64 \mathrm{~mL}, 4.40 \mathrm{mmol})$ and $(R)$ binol $(286 \mathrm{mg}, 1.00 \mathrm{mmol})$ were subsequently added and the solution was left to stir overnight. The volatiles were removed in vacuo and the crude product was filtered through a plug of silica in toluene. The title product was obtained after removal of the solvent as a white solid. Yield: $404 \mathrm{mg}(71 \%)$. MP: $158{ }^{\circ} \mathrm{C}$. $[\alpha]_{\mathrm{D}}^{20}=+238^{\circ}\left(c=1.0 \mathrm{mg} \mathrm{mL}^{-1}, \mathrm{CHCl}_{3}\right)$. IR (neat): $v$ 3055.4, 2981.3, 1588.6, 1505.9, 1462.5, 1362.2, 1326.4, 1228.2, 1203.1, $1154.9,1070.0,949.2,869.5,818.8,782.0,747.7,629.4 \mathrm{~cm}^{-1}$. ${ }^{1} \mathrm{H}$ NMR $\left(\mathrm{CD}_{2} \mathrm{Cl}_{2}, 500 \mathrm{MHz}\right): \delta(\mathrm{ppm}) 8.06\left(\mathrm{~d},{ }^{3} J_{\mathrm{HH}}=8.3 \mathrm{~Hz}\right.$, $\left.1 \mathrm{H}, H 4^{\prime}\right), 8.01\left(\mathrm{~d},{ }^{3} J_{\mathrm{HH}}=8.3 \mathrm{~Hz}, 1 \mathrm{H}, \operatorname{Ar} H\right), 7.95-7.91(\mathrm{~m}, 3 \mathrm{H}$, $H 14 / \operatorname{Ar} H), 7.88\left(\mathrm{~d},{ }^{3} J_{\mathrm{HH}}=8.2 \mathrm{~Hz}, 1 \mathrm{H}, \operatorname{Ar} H\right), 7.84\left(\mathrm{dd},{ }^{3} J_{\mathrm{HH}}=\right.$ $\left.7.0 \mathrm{~Hz},{ }^{5} J_{\mathrm{HP}}=1.0 \mathrm{~Hz}, 1 \mathrm{H}, H 2^{\prime}\right), 7.73\left(\mathrm{~d},{ }^{3} J_{\mathrm{HH}}=8.7 \mathrm{~Hz}, 1 \mathrm{H}\right.$, $\left.H 14^{\prime}\right), 7.69\left(\mathrm{dd},{ }^{3} J_{\mathrm{HH}}=8.3 \mathrm{~Hz},{ }^{3} J_{\mathrm{HH}}=7.0 \mathrm{~Hz}, 1 \mathrm{H}, H 3^{\prime}\right), 7.61$ $\left(\mathrm{d},{ }^{3} J_{\mathrm{HH}}=8.6 \mathrm{~Hz}, 1 \mathrm{H}, H 4\right), 7.56-7.52,(\mathrm{~m}, 2 \mathrm{H}, \mathrm{ArH}), 7.49-7.40$ (m, 4H, H13/ArH), 7.38-7.31 (m, 5H, ArH), 7.30-7.24 (m, 3H, $H 3 / \mathrm{Ar} H), 6.91\left(\mathrm{~d},{ }^{3} J_{\mathrm{HH}}=8.7 \mathrm{~Hz}, 1 \mathrm{H}, H 13^{\prime}\right) .{ }^{13} \mathrm{C}\left\{{ }^{1} \mathrm{H}\right\}$ NMR 
$\left(\mathrm{CD}_{2} \mathrm{Cl}_{2}, 126 \mathrm{MHz}\right): \delta(\mathrm{ppm}) 150.2\left(\mathrm{~d},{ }^{2} J_{\mathrm{CP}}=2.4 \mathrm{~Hz}, C 12\right)$, $149.0\left(\mathrm{~d},{ }^{2} J_{\mathrm{CP}}=6.1 \mathrm{~Hz}, C 12^{\prime}\right), 145.0\left(\mathrm{~d},{ }^{2} J_{\mathrm{CP}}=37.2 \mathrm{~Hz}, C 1\right)$, $136.2\left(\mathrm{~d},{ }^{1} J_{\mathrm{CP}}=38.9 \mathrm{~Hz}, C 2\right), 135.0,134.8\left(\mathrm{~d}, J_{\mathrm{CP}}=10.0 \mathrm{~Hz}\right)$, $133.5,133.3\left(\mathrm{~d}, J_{\mathrm{CP}}=2.1 \mathrm{~Hz}\right), 133.0\left(\mathrm{~d}, J_{\mathrm{CP}}=4.7 \mathrm{~Hz}\right), 132.9$ $\left(\mathrm{d}, J_{\mathrm{CP}}=1.5 \mathrm{~Hz}\right), 132.8\left(\mathrm{~d}, J_{\mathrm{CP}}=1.0 \mathrm{~Hz}\right), 131.7,131.2,130.9$ $\left(\mathrm{d},{ }^{4} J_{\mathrm{CP}}=5.8 \mathrm{~Hz}, C 2^{\prime}\right), 130.6(C 14), 129.6\left(\mathrm{~d},{ }^{4} J_{\mathrm{CP}}=0.7 \mathrm{~Hz}\right.$, $\left.C^{\prime} 4^{\prime}\right), 129.1\left(C 4^{\prime}\right), 128.5,128.4,128.3,128.2,127.7,127.1$ $(C 4), 127.0\left(\mathrm{~d}, J_{\mathrm{CP}}=2.8 \mathrm{~Hz}\right), 126.8,126.7,126.6,126.6,126.5$, $126.3,126.3,126.2,125.0,124.9\left(C 3^{\prime}\right), 124.9,124.7\left(\mathrm{~d},{ }^{3} J_{\mathrm{CP}}=\right.$ $5.7 \mathrm{~Hz}, C 11), 124.2\left(\mathrm{~d},{ }^{2} J_{\mathrm{CP}}=2.7 \mathrm{~Hz}, C 3\right), 123.8\left(\mathrm{~d},{ }^{3} J_{\mathrm{CP}}=2.6\right.$ $\left.\mathrm{Hz}, C 11^{\prime}\right), 122.2\left(C 13^{\prime}\right), 121.5\left(\mathrm{~d},{ }^{3} J_{\mathrm{CP}}=1.4 \mathrm{~Hz}, C 13\right) .{ }^{31} \mathrm{P}\left\{{ }^{1} \mathrm{H}\right\}$ NMR $\left(\mathrm{CD}_{2} \mathrm{Cl}_{2}, 202 \mathrm{MHz}\right): \delta$ (ppm) 177.4. HRMS ( $\mathrm{m} / z, \mathrm{ESI}^{+}$, $\mathrm{MeOH})$ : found 585.1605 , calcd for $\left[\mathrm{M}+\mathrm{H}_{2} \mathrm{O}\right]^{+} 585.1614$.

\section{Synthesis of $\left(S, S_{\mathrm{b}}\right)$-[1,1'-binaphthalene]-2,2'-diyl [1,1'-binaphthalen]-2-ylphosphonite (5b)}

The same procedure was followed as for 5a, except for using $(S)$-binol as the nucleophile. The title product was obtained as a white solid after removal of the solvent. Yield: $527 \mathrm{mg}(93 \%)$. MP: $197{ }^{\circ} \mathrm{C}$. $[\alpha]_{\mathrm{D}}^{20}=-264^{\circ}\left(c=1.0 \mathrm{mg} \mathrm{mL}{ }^{-1}, \mathrm{CHCl}_{3}\right)$. IR (neat): $v 3060.6,2981.3,1588.6,1506.5,1466.0,1366.0$, 1330.0, 1262.7, 1232.0, 1204.0, 1141.6, 1141.6, 1072.2, 951.6, $869.5,822.7,789.4, \quad 753.7,684.6,630.2 \mathrm{~cm}^{-1} .{ }^{1} \mathrm{H}$ NMR $\left(\mathrm{CD}_{2} \mathrm{Cl}_{2}, 500 \mathrm{MHz}\right): \delta(\mathrm{ppm}) 8.04\left(\mathrm{~d},{ }^{3} J_{\mathrm{HH}}=8.2 \mathrm{~Hz}, 1 \mathrm{H}, H 4^{\prime}\right)$, 7.99-7.89 (m, 4H, H14'/ArH), $7.88\left(\mathrm{~d},{ }^{3} J_{\mathrm{HH}}=8.2 \mathrm{~Hz}, 1 \mathrm{H}, H 5\right)$, $7.78\left(\mathrm{~d},{ }^{3} J_{\mathrm{HH}}=8.7 \mathrm{~Hz}, 1 \mathrm{H}, H 14\right), 7.68\left(\mathrm{dd},{ }^{3} J_{\mathrm{HH}}=8.2 \mathrm{~Hz},{ }^{3} J_{\mathrm{HH}}\right.$ $\left.=7.0 \mathrm{~Hz}, 1 \mathrm{H}, H 3^{\prime}\right), 7.63\left(\mathrm{dd},{ }^{3} J_{\mathrm{HH}}=7.0 \mathrm{~Hz},{ }^{5} J_{\mathrm{HP}}=1.2 \mathrm{~Hz}, 1 \mathrm{H}\right.$, $\left.H 2^{\prime}\right), 7.61\left(\mathrm{~d},{ }^{3} J_{\mathrm{HH}}=8.6 \mathrm{~Hz}, 1 \mathrm{H}, H 4\right), 7.55\left(\mathrm{ddd},{ }^{3} J_{\mathrm{HH}}=8.2 \mathrm{~Hz}\right.$, $\left.{ }^{3} J_{\mathrm{HH}}=5.5 \mathrm{~Hz},{ }^{4} J_{\mathrm{HH}}=2.5 \mathrm{~Hz}, 1 \mathrm{H}, H 6\right), 7.53-7.36(\mathrm{~m}, 7 \mathrm{H}$, $\left.H 13^{\prime} / \mathrm{ArH}\right), 7.35-7.28$ (m, 3H, $\left.H 7 / H 8 / \mathrm{Ar} H\right), 7.23-7.21$ (m, 3H, $H 3 / \mathrm{ArH}), 6.90\left(\mathrm{~d},{ }^{3} J_{\mathrm{HH}}=8.7 \mathrm{~Hz}, 1 \mathrm{H}, H 13\right) .{ }^{13} \mathrm{C}\left\{{ }^{1} \mathrm{H}\right\} \mathrm{NMR}$ $\left(\mathrm{CD}_{2} \mathrm{Cl}_{2}, 126 \mathrm{MHz}\right): \delta$ (ppm) $150.1\left(\mathrm{~d},{ }^{2} J_{\mathrm{CP}}=1.9 \mathrm{~Hz}, C 12^{\prime}\right)$, $148.9\left(\mathrm{~d},{ }^{2} J_{\mathrm{CP}}=5.7 \mathrm{~Hz}, C 12\right), 145.4\left(\mathrm{~d},{ }^{2} J_{\mathrm{CP}}=37.0 \mathrm{~Hz}, C 1\right)$, $136.5\left(\mathrm{~d},{ }^{1} J_{\mathrm{CP}}=40.4 \mathrm{~Hz}, C 2\right), 135.0\left(\mathrm{~d}, J_{\mathrm{CP}}=7.9 \mathrm{~Hz}\right), 134.1(\mathrm{~d}$, $\left.J_{\mathrm{CP}}=2.9 \mathrm{~Hz}\right), 133.5,133.0\left(\mathrm{~d}, J_{\mathrm{CP}}=4.3 \mathrm{~Hz}\right), 132.9,132.8$, 131.6, 131.4, 131.2, $130.6\left(C 14^{\prime}\right), 129.6(C 14), 129.4\left(C 2^{\prime}\right)$, 128.7 (C4'), 128.5, 128.4, 128.4, 128.1 (C5), 127.8, 127.4 (d, $\left.J_{\mathrm{CP}}=2.6 \mathrm{~Hz}\right), 127.1(C 4), 127.0,126.9,126.8,126.6,126.5$, $126.4,126.2,126.1,125.9,125.1\left(C 3^{\prime}\right), 124.9,124.8\left(C 11^{\prime}\right)$, $124.3\left(\mathrm{~d},{ }^{2} J_{\mathrm{CP}}=2.6 \mathrm{~Hz}, C 3\right), 123.6(C 11), 122.5(C 13), 121.4$ $\left(C 13^{\prime}\right) .{ }^{31} \mathrm{P}\left\{{ }^{1} \mathrm{H}\right\}$ NMR $\left(\mathrm{CD}_{2} \mathrm{Cl}_{2}, 202 \mathrm{MHz}\right): \delta(\mathrm{ppm})$ 175.7. HRMS $\left(\mathrm{m} / z, \mathrm{EI}^{+}\right)$: found: $=567.1514$, calcd for $[\mathrm{M}-\mathrm{H}]^{+}$ 567.1508 .

\section{Synthesis of $\left(R, R_{\mathrm{b}}\right)-\left[1,1^{\prime}\right.$-binaphthalene]-2,2'-diyl (2'-methoxy-[1,1'-binaphthalen]-2-yl)phosphonite (6a)}

$\mathrm{PCl}_{5}$ (458 mg, $2.20 \mathrm{mmol}$ ) was dissolved in toluene $(8 \mathrm{~mL}) .(R)-$ $2(316 \mathrm{mg}, 1.00 \mathrm{mmol})$ was added and the reaction mixture was left to stir for $45 \mathrm{~min}$. The volatiles were removed in vacuo to give $(R)-4\left({ }^{31} \mathrm{P}\left\{{ }^{1} \mathrm{H}\right\} \mathrm{NMR}, \mathrm{CDCl}_{3}: \delta=159.1 \mathrm{ppm}\right)$ as a yellow solid. THF ( $8 \mathrm{~mL}), \mathrm{NEt}_{3}(448 \mathrm{mg}, 0.64 \mathrm{~mL}, 4.40 \mathrm{mmol})$ and $(R)$-binol (286 mg, $1.00 \mathrm{mmol})$ were added subsequently and the solution was left to stir overnight. The volatiles were removed in vacuo and the crude product was dissolved in toluene and filtered through a plug of silica. The title product was obtained after removal of the solvent as a white solid. Yield: $523 \mathrm{mg}$,
(87\%). MP: $>270{ }^{\circ} \mathrm{C} .[\alpha]_{\mathrm{D}}^{20}=+444^{\circ}\left(c=1.0 \mathrm{mg} \mathrm{mL}^{-1}, \mathrm{CHCl}_{3}\right)$. IR (neat): $v$ 2981.2, 1619.8, 1590.0, 1507.0, 1461.8, 1431.0, $1327.7,1228.2,1149.5,1078.1,947.3,866.6,820.5,799.5$, 746.7, 686.7, $630.3 \mathrm{~cm}^{-1} .{ }^{1} \mathrm{H}$ NMR $\left(\mathrm{CD}_{2} \mathrm{Cl}_{2}, 500 \mathrm{MHz}\right): \delta$ (ppm) $8.09\left(\mathrm{~d},{ }^{3} J_{\mathrm{HH}}=9.1 \mathrm{~Hz}, 1 \mathrm{H}, H 4^{\prime}\right), 7.94-7.90\left(\mathrm{~m}, 4 \mathrm{H}, H 5^{\prime}\right.$ $\left.H 14 / H 15^{\prime} / \mathrm{ArH}\right), 7.89\left(\mathrm{~d},{ }^{3} J_{\mathrm{HH}}=8.2 \mathrm{~Hz}, 1 \mathrm{H}, H 5\right), 7.72\left(\mathrm{~d},{ }^{3} J_{\mathrm{HH}}\right.$ $\left.=8.8 \mathrm{~Hz}, 1 \mathrm{H}, H 14^{\prime}\right), 7.59\left(\mathrm{~d},{ }^{3} J_{\mathrm{HH}}=8.5 \mathrm{~Hz}, 1 \mathrm{H}, H 4\right), 7.55$ $\left(\mathrm{ddd},{ }^{3} J_{\mathrm{HH}}=8.2 \mathrm{~Hz},{ }^{3} J_{\mathrm{HH}}=5.7 \mathrm{~Hz},{ }^{4} J_{\mathrm{HH}}=2.1 \mathrm{~Hz}, 1 \mathrm{H}, H 6\right)$, $7.53\left(\mathrm{~d},{ }^{3} J_{\mathrm{HH}}=9.1 \mathrm{~Hz}, 1 \mathrm{H}, H 3^{\prime}\right), 7.47\left(\mathrm{ddd},{ }^{3} J_{\mathrm{HH}}=8.2 \mathrm{~Hz}\right.$, $\left.{ }^{3} J_{\mathrm{HH}}=6.8 \mathrm{~Hz},{ }^{4} J_{\mathrm{HH}}=1.2 \mathrm{~Hz}, 1 \mathrm{H}, \operatorname{Ar} H\right), 7.44-7.40(\mathrm{~m}, 3 \mathrm{H}$, $H 13 / \mathrm{Ar} H), 7.38\left(\mathrm{ddd},{ }^{3} J_{\mathrm{HH}}=8.2 \mathrm{~Hz},{ }^{3} J_{\mathrm{HH}}=6.8 \mathrm{~Hz},{ }^{4} J_{\mathrm{HH}}=1.2\right.$ $\mathrm{Hz}, 1 \mathrm{H}, \mathrm{ArH}), 7.36-7.24(\mathrm{~m}, 6 \mathrm{H}, \mathrm{ArH}), 7.23\left(\mathrm{dd},{ }^{3} J_{\mathrm{HH}}=8.5\right.$ $\left.\mathrm{Hz},{ }^{3} J_{\mathrm{HP}}=1.4 \mathrm{~Hz}, 1 \mathrm{H}, H 3\right), 7.02\left(\mathrm{~d},{ }^{3} J_{\mathrm{HH}}=8.5 \mathrm{~Hz}, 1 \mathrm{H}, \mathrm{ArH}\right)$, $6.93\left(\mathrm{~d},{ }^{3} J_{\mathrm{HH}}=8.8 \mathrm{~Hz}, 1 \mathrm{H}, H 13\right.$ '), $3.99\left(\mathrm{~s}, 3 \mathrm{H}, \mathrm{OCH}_{3}\right) .{ }^{13} \mathrm{C}\left\{{ }^{1} \mathrm{H}\right\}$ NMR $\left(\mathrm{CD}_{2} \mathrm{Cl}_{2}, 126 \mathrm{MHz}\right): \delta(\mathrm{ppm}) 156.6\left(\mathrm{~d},{ }^{4} J_{\mathrm{CP}}=3.5 \mathrm{~Hz}\right.$, $\left.C 2^{\prime}\right), 150.3\left(\mathrm{~d},{ }^{2} J_{\mathrm{CP}}=2.5 \mathrm{~Hz}, C 12\right), 154.9\left(\mathrm{~d},{ }^{2} J_{\mathrm{CP}}=5.8 \mathrm{~Hz}\right.$, $\left.C 12^{\prime}\right), 141.8\left(\mathrm{~d},{ }^{2} J_{\mathrm{CP}}=37.5 \mathrm{~Hz}, C 1\right), 136.3\left(\mathrm{~d},{ }^{1} J_{\mathrm{CP}}=37.7 \mathrm{~Hz}\right.$, $C 2), 135.3,134.5\left(\mathrm{~d},{ }^{4} J_{\mathrm{CP}}=2.9 \mathrm{~Hz}, C 9^{\prime}\right), 132.9,132.8,132.7$ $\left(\mathrm{d}, J_{\mathrm{CP}}=1.0 \mathrm{~Hz}\right), 131.6\left(\mathrm{~d}, J_{\mathrm{CP}}=0.9 \mathrm{~Hz}\right), 131.4,131.2,130.8$ $\left(C 4^{\prime}\right), 130.5\left(C 5^{\prime}\right), 129.5\left(C 14^{\prime}\right), 128.7,128.5,128.5,128.3$ (C5), $128.2\left(C 10^{\prime}\right), 128.1,127.7,127.1,126.8,126.7(C 4)$, $126.6,126.5,126.4\left(\mathrm{~d}, J_{\mathrm{CP}}=2.6 \mathrm{~Hz}\right), 126.2,126.1,125.2$, $124.9,124.8,124.5\left(\mathrm{~d},{ }^{2} J_{\mathrm{CP}}=2.0 \mathrm{~Hz}, C 3\right), 123.8,122.7(C 13)$, $121.6\left(C 13^{\prime}\right), 118.9\left(\mathrm{~d},{ }^{3} J_{\mathrm{CP}}=10.2 \mathrm{~Hz}, C 1^{\prime}\right), 112.8\left(C 3^{\prime}\right), 56.2$ $\left(\mathrm{s}, \mathrm{OCH}_{3}\right) .{ }^{31} \mathrm{P}\left\{{ }^{1} \mathrm{H}\right\} \mathrm{NMR}\left(\mathrm{CD}_{2} \mathrm{Cl}_{2}, 202 \mathrm{MHz}\right): \delta(\mathrm{ppm}) 177.8$. HRMS $\left(m / z, \mathrm{ESI}^{+}, \mathrm{CH}_{2} \mathrm{Cl}_{2}\right)$ : found 599.1767, calcd for $[\mathrm{M}+\mathrm{H}]^{+}$599.1771.

\section{Synthesis of $\left(R, S_{\mathrm{b}}\right)-\left[1,1^{\prime}\right.$-binaphthalene]-2,2'-diyl (2'-methoxy-[1,1'-binaphthalen]-2-yl)phosphonite (6b)}

The same procedure was followed as for $\mathbf{6 a}$, except for using $(S)$-binol as the nucleophile. The title product was obtained as a white solid after removal of the solvent. Yield: $430 \mathrm{mg}(72 \%)$. MP: $231{ }^{\circ} \mathrm{C}$ (decomposition). $[\alpha]_{\mathrm{D}}^{20}=-310^{\circ}\left(c=1.0 \mathrm{mg} \mathrm{mL}^{-1}\right.$, $\mathrm{CHCl}_{3}$ ). IR (neat): $v$ 2980.8, 1620.1, 1590.2, 1506.3, 1462.7, $1431.5,1329.8,1230.2,1203.4,1146.0,1070.1,949.5,867.8$, 820.8, 789.7, 747.2, 684.7, $628.8 \mathrm{~cm}^{-1}$. ${ }^{1} \mathrm{H}$ NMR $\left(\mathrm{CD}_{2} \mathrm{Cl}_{2}\right.$, $500 \mathrm{MHz}): \delta(\mathrm{ppm}) 8.09\left(\mathrm{~d},{ }^{3} J_{\mathrm{HH}}=9.1 \mathrm{~Hz}, 1 \mathrm{H}, H 4^{\prime}\right), 7.97(\mathrm{~d}$, $\left.{ }^{3} J_{\mathrm{HH}}=8.8 \mathrm{~Hz}, 1 \mathrm{H}, H 14^{\prime}\right), 7.93-7.90\left(\mathrm{~m}, 3 \mathrm{H}, H 15 / H 5^{\prime} / H 15^{\prime}\right)$, $7.88\left(\mathrm{~d},{ }^{3} J_{\mathrm{HH}}=8.2 \mathrm{~Hz}, 1 \mathrm{H}, H 5\right), 7.76\left(\mathrm{~d},{ }^{3} J_{\mathrm{HH}}=8.7 \mathrm{~Hz}, 1 \mathrm{H}\right.$, H14), $7.60\left(\mathrm{~d},{ }^{3} J_{\mathrm{HH}}=8.6 \mathrm{~Hz}, 1 \mathrm{H}, H 4\right), 7.54\left(\mathrm{dd},{ }^{3} J_{\mathrm{HH}}=8.0 \mathrm{~Hz}\right.$, $\left.{ }^{3 / 4} J_{\mathrm{HH}}=4.0 \mathrm{~Hz}, 1 \mathrm{H}, H 6^{\prime}\right), 7.52\left(\mathrm{~d},{ }^{3} J_{\mathrm{HH}}=8.8 \mathrm{~Hz}, 1 \mathrm{H}, H 13^{\prime}\right)$, $7.51\left(\mathrm{~d},{ }^{3} J_{\mathrm{HH}}=9.1 \mathrm{~Hz}, 1 \mathrm{H}, H 3^{\prime}\right), 7.46\left(\mathrm{ddd},{ }^{3} J_{\mathrm{HH}}=8.0 \mathrm{~Hz}\right.$, $\left.{ }^{3} J_{\mathrm{HH}}=6.8 \mathrm{~Hz},{ }^{4} J_{\mathrm{HH}}=1.0 \mathrm{~Hz}, 1 \mathrm{H}, H 16\right), 7.42-7.35(\mathrm{~m}, 4 \mathrm{H}$, $H 18 / \mathrm{Ar} H$ ), 7.32 (d, ${ }^{3} J_{\mathrm{HH}}=4.0 \mathrm{~Hz}, 2 \mathrm{H}, H 7^{\prime} / H 8^{\prime}$ ), 7.30 (ddd, $\left.{ }^{3} J_{\mathrm{HH}}=8.4 \mathrm{~Hz},{ }^{3} J_{\mathrm{HH}}=6.8 \mathrm{~Hz},{ }^{4} J_{\mathrm{HH}}=1.3 \mathrm{~Hz}, 1 \mathrm{H}, H 17\right)$, $7.23-7.18(\mathrm{~m}, 4 \mathrm{H}, H 3 / \mathrm{Ar} H), 6.87\left(\mathrm{~d},{ }^{3} J_{\mathrm{HH}}=8.7 \mathrm{~Hz}, 1 \mathrm{H}, H 13\right)$, $3.89\left(\mathrm{~s}, 3 \mathrm{H}, \mathrm{OCH}_{3}\right) \cdot{ }^{13} \mathrm{C}\left\{{ }^{1} \mathrm{H}\right\} \mathrm{NMR}\left(\mathrm{CD}_{2} \mathrm{Cl}_{2}, 101 \mathrm{MHz}\right): \delta$ (ppm) $154.9\left(\mathrm{~d},{ }^{4} J_{\mathrm{CP}}=2.9 \mathrm{~Hz}, C 2^{\prime}\right), 150.2\left(\mathrm{~d},{ }^{2} J_{\mathrm{CP}}=2.3 \mathrm{~Hz}\right.$, $\left.C 12^{\prime}\right), 149.0\left(\mathrm{~d},{ }^{2} J_{\mathrm{CP}}=5.9 \mathrm{~Hz}, C 12\right), 141.9\left(\mathrm{~d},{ }^{2} J_{\mathrm{CP}}=39.2 \mathrm{~Hz}\right.$, $C 1), 136.4\left(\mathrm{~d},{ }^{1} J_{\mathrm{CP}}=38.3 \mathrm{~Hz}, C 2\right), 135.3,135.0\left(C 9^{\prime}\right), 132.8$, $132.7,132.7,131.6,131.1,130.7\left(C 4^{\prime}\right), 130.5\left(C 14^{\prime}\right), 129.5$ (C14), 128.8, 128.4 (C15), $128.4\left(C 15^{\prime}\right), 128.3\left(C 5^{\prime}\right), 128.3$ $\left(C 10^{\prime}\right), 128.2(C 5), 127.7,126.9(C 4), 126.8,126.7,126.6$, $126.5,126.4\left(\mathrm{~d}, J_{\mathrm{CP}}=2.1 \mathrm{~Hz}\right), 126.2,126.1,125.5,124.9$, $124.8(C 16), 124.6\left(\mathrm{~d}, J_{\mathrm{CP}}=2.5 \mathrm{~Hz}\right), 123.7,123.6\left(\mathrm{~d}, J_{\mathrm{CP}}=2.5\right.$ $\mathrm{Hz}), 122.5$ (C13), $121.5\left(C 13^{\prime}\right), 119.0\left(\mathrm{~d},{ }^{3} J_{\mathrm{CP}}=10.3 \mathrm{~Hz}, C 1^{\prime}\right)$, 
$113.0 \quad\left(C 3^{\prime}\right), \quad 56.4 \quad\left(\mathrm{~s}, \quad \mathrm{OCH}_{3}\right) .{ }^{31} \mathrm{P}\left\{{ }^{1} \mathrm{H}\right\} \quad \mathrm{NMR} \quad\left(\mathrm{CD}_{2} \mathrm{Cl}_{2}\right.$, $202 \mathrm{MHz}$ ): $\delta$ (ppm) 177.9. HRMS ( $m / z, \mathrm{ESI}^{+}, \mathrm{CH}_{2} \mathrm{Cl}_{2}$ ): found 599.1766, calcd for $[\mathrm{M}+\mathrm{H}]^{+} 599.1771$.

\section{Synthesis of $\left[\mathrm{Pd}(5 \mathrm{a})\left(\eta^{3}-\mathrm{C}_{4} \mathrm{H}_{7}\right) \mathrm{Cl}\right](7 \mathrm{a})$}

$\left[\operatorname{Pd}\left(\eta^{3}-\mathrm{C}_{4} \mathrm{H}_{7}\right) \mathrm{Cl}\right]_{2}(7 \mathrm{mg}, 18 \mu \mathrm{mol})$ and 5a $(20 \mathrm{mg}, 35 \mu \mathrm{mol})$ were dissolved in $\mathrm{CH}_{2} \mathrm{Cl}_{2}(1 \mathrm{~mL})$ and stirred for $10 \mathrm{~min}$. The intended complex was formed quantitatively. Slow diffusion of diethyl ether into the reaction mixture yielded colorless crystals overnight, which were suitable for X-ray diffraction analysis. Yield: $25 \mathrm{mg}$ (92\%). MP: $>270{ }^{\circ} \mathrm{C}$. IR (neat): $v$ 3052.0, 1587.6, 1505.8, 1460.0, 1432.9, 1360.3, 1322.4, 1220.7, 1067.8, 977.3, $942.2, \quad 871.8, \quad 838.4, \quad 810.2, \quad 759.4, \quad 706.9, \quad 677.2,634.3$, $596.8 \mathrm{~cm}^{-1}$. ${ }^{1} \mathrm{H}$ NMR $\left(\mathrm{CD}_{2} \mathrm{Cl}_{2}, 500 \mathrm{MHz}\right): \delta(\mathrm{ppm})$ isomer A (97\%), $7.97\left(\mathrm{~d},{ }^{3} J_{\mathrm{HH}}=8.1 \mathrm{~Hz}, 1 \mathrm{H}, H 4^{\prime}\right), 7.94-7.90(\mathrm{~m}, 4 \mathrm{H}$, $\left.H 14 / H 5 / H 15 / H 15^{\prime}\right), 7.89-7.85$ (m, 2H, H2-/H5'), 7.84-7.80 (m, $2 \mathrm{H}, H 13 / H 4), 7.78\left(\mathrm{~d},{ }^{3} J_{\mathrm{HH}}=8.9 \mathrm{~Hz}, 1 \mathrm{H}, H 14^{\prime}\right), 7.72\left(\mathrm{dd},{ }^{3} J_{\mathrm{HH}}\right.$ $\left.=8.6 \mathrm{~Hz},{ }^{3} J_{\mathrm{HP}}=5.5 \mathrm{~Hz}, 1 \mathrm{H}, H 3\right), 7.69\left(\mathrm{dd},{ }^{3} J_{\mathrm{HH}}=8.1 \mathrm{~Hz},{ }^{3} J_{\mathrm{HH}}\right.$ $\left.=7.2 \mathrm{~Hz}, 1 \mathrm{H}, H 3^{\prime}\right), 7.58-7.55(\mathrm{~m}, 1 \mathrm{H}, \mathrm{Ar} H), 7.53-7.43(\mathrm{~m}, 5 \mathrm{H}$, $H 6^{\prime} / \mathrm{ArH}$ ), 7.39-7.27 (m, 5H, ArH), 7.18-7.13 (m, 2H, H13'/ $\mathrm{Ar} H), 3.75\left(\mathrm{dd},{ }^{3} J_{\mathrm{HP}}=9.9 \mathrm{~Hz},{ }^{4} J_{\mathrm{HH}}=2.5 \mathrm{~Hz}, 1 \mathrm{H}\right.$, allyl $\left.-H \mathrm{t}_{\mathrm{syn}}\right)$, $2.56\left(\mathrm{~s}, 1 \mathrm{H}\right.$, allyl- $\left.H \mathrm{c}_{\mathrm{syn}}\right), 1.57\left(\mathrm{~d},{ }^{3} J_{\mathrm{HP}}=14.1 \mathrm{~Hz}, 1 \mathrm{H}\right.$, allyl$\left.H \mathrm{t}_{\text {anti }}\right), 1.00\left(\mathrm{~s}, 3 \mathrm{H}\right.$, allyl- $\left.\mathrm{CH}_{3}\right), 0.83\left(\mathrm{~s}, 1 \mathrm{H}\right.$, allyl $\left.-H \mathrm{c}_{\text {anti }}\right)$; isomer B (3\%), 8.00-6.99 (m, 23H, ArH), $6.92(\mathrm{~m}, 1 \mathrm{H}, \mathrm{ArH}), 6.87$ (m, $1 \mathrm{H}, \mathrm{Ar} H), 3.96\left(\mathrm{~d},{ }^{3} J_{\mathrm{HP}}=6.8 \mathrm{~Hz}, 1 \mathrm{H}\right.$, allyl- $\left.H \mathrm{t}_{\mathrm{syn}}\right), 2.96(\mathrm{~m}, 1 \mathrm{H}$, allyl- $\left.H \mathrm{c}_{\mathrm{syn}}\right), 2.64\left(\mathrm{~d},{ }^{3} J_{\mathrm{HP}}=12.3 \mathrm{~Hz}, 1 \mathrm{H}\right.$, allyl- $\left.H \mathrm{t}_{\mathrm{anti}}\right), 1.53(\mathrm{~m}$, $1 \mathrm{H}$, allyl- $\left.\mathrm{Hc}_{\text {anti }}\right), 1.42$ (s, 3H, allyl-C $\left.H_{3}\right) .{ }^{13} \mathrm{C}\left\{{ }^{1} \mathrm{H}\right\}$ NMR $\left(\mathrm{CD}_{2} \mathrm{Cl}_{2}, 126 \mathrm{MHz}\right): \delta(\mathrm{ppm})$ isomer A $(97 \%), 149.1\left(\mathrm{~d},{ }^{2} J_{\mathrm{CP}}=\right.$ $5.3 \mathrm{~Hz}, C 12), 148.6\left(\mathrm{~d},{ }^{2} J_{\mathrm{CP}}=13.2 \mathrm{~Hz}, C 122^{\prime}\right), 144.7\left(\mathrm{~d},{ }^{2} J_{\mathrm{CP}}=\right.$ $27.2 \mathrm{~Hz}, C 1), 135.2,134.7\left(\mathrm{~d},{ }^{1} J_{\mathrm{CP}}=9.3 \mathrm{~Hz}, C 2\right), 133.5,133.4$, $133.3,133.1,132.6\left(\mathrm{~d}, J_{\mathrm{CP}}=1.4 \mathrm{~Hz}\right), 132.2\left(\mathrm{~d}, J_{\mathrm{CP}}=1.9 \mathrm{~Hz}\right)$, $131.9\left(\mathrm{~d}, J_{\mathrm{CP}}=1.3 \mathrm{~Hz}\right), 131.5\left(\mathrm{~d}, J_{\mathrm{CP}}=1.3 \mathrm{~Hz}\right), 131.1\left(C 2^{\prime}\right)$, $131.0\left(\mathrm{~d}, J_{\mathrm{CP}}=28.6 \mathrm{~Hz}\right.$, allyl-C), $130.7\left(\mathrm{~d},{ }^{4} J_{\mathrm{CP}}=1.1 \mathrm{~Hz}, C 14\right)$, $130.2\left(\mathrm{~d},{ }^{4} J_{\mathrm{CP}}=1.4 \mathrm{~Hz}, C 14^{\prime}\right), 129.7,129.2\left(C 4^{\prime}\right), 128.6,128.5$, $128.3,128.2,128.2,127.7\left(\mathrm{~d},{ }^{3} J_{\mathrm{CP}}=5.2 \mathrm{~Hz}, C 4\right), 127.3,127.1$, $127.1,127.0\left(\mathrm{~d}, J_{\mathrm{CP}}=2.3 \mathrm{~Hz}\right), 126.9,126.8,126.7,126.6$, $126.4,125.5,125.4,125.2\left(C 3^{\prime}\right), 124.9\left(\mathrm{~d},{ }^{2} J_{\mathrm{CP}}=2.8 \mathrm{~Hz}, C 3\right)$, $124.2\left(\mathrm{~d},{ }^{3} J_{\mathrm{CP}}=3.8 \mathrm{~Hz}, C 11\right), 123.7\left(\mathrm{~d},{ }^{3} J_{\mathrm{CP}}=2.9 \mathrm{~Hz}, C 11^{\prime}\right)$, $122.4\left(\mathrm{~d},{ }^{3} J_{\mathrm{CP}}=2.4 \mathrm{~Hz}, C 13\right), 120.4\left(C 13^{\prime}\right), 76.8\left(\mathrm{~d},{ }^{2} J_{\mathrm{CP}}=45.3\right.$ $\mathrm{Hz}$, allyl-Ct), 56.1 (d, ${ }^{2} J_{\mathrm{CP}}=5.8 \mathrm{~Hz}$, allyl-Cc), 22.4 (allyl- $\mathrm{CH}_{3}$ ); signals of isomer B could not be observed. ${ }^{31} \mathrm{P}\left\{{ }^{1} \mathrm{H}\right\}$ NMR $\left(\mathrm{CD}_{2} \mathrm{Cl}_{2}, 202 \mathrm{MHz}\right): \delta(\mathrm{ppm})$ isomer A $(97 \%), 173.4$; isomer B (3\%), 175.7. HRMS ( $\left.m / z, \mathrm{ESI}^{+}, \mathrm{MeOH}\right)$ : found 727.1178, calcd for $[\mathrm{M}-\mathrm{Cl}]^{+} 727.1176$.

\section{Synthesis of $\left[\operatorname{Pd}(5 b)\left(\eta^{3}-\mathrm{C}_{4} \mathrm{H}_{7}\right) \mathrm{Cl}\right](7 \mathrm{~b})$}

The same procedure was followed as for 7a. Yield: $23 \mathrm{mg}$ (83\%). MP: $>270{ }^{\circ} \mathrm{C}$. IR (neat): $v$ 3047.7, 1585.8, 1505.6, 1461.1, 1434.2, 1360.9, 1322.9, 1269.3, 1222.1, 1161.0, 1121.5, 1068.7, 1027.5, 977.3, 943.4, 877.1, 839.5, 803.3, 757.5, 729.6, 687.3, 634.8, 598.0, 557.6 $\mathrm{cm}^{-1} .{ }^{1} \mathrm{H}$ NMR $\left(\mathrm{CD}_{2} \mathrm{Cl}_{2}, 500 \mathrm{MHz}\right)$ : $\delta(\mathrm{ppm}) 8.13\left(\mathrm{~d},{ }^{3} J_{\mathrm{HH}}=7.0 \mathrm{~Hz}, 1 \mathrm{H}, H 2^{\prime}\right), 7.98-7.86(\mathrm{~m}, 7 \mathrm{H}$, $\left.H 4^{\prime} / H 15 / H 5^{\prime} / H 14^{\prime} / H 5 / H 15^{\prime} / H 13^{\prime}\right), 7.83\left(\mathrm{~d},{ }^{3} J_{\mathrm{HH}}=8.8 \mathrm{~Hz}, 1 \mathrm{H}\right.$, $H 14), 7.74\left(\mathrm{~d},{ }^{3} J_{\mathrm{HH}}=8.7 \mathrm{~Hz}, 1 \mathrm{H}, H 4\right), 7.69\left(\mathrm{dd},{ }^{3} J_{\mathrm{HH}}=8.7 \mathrm{~Hz}\right.$, $\left.{ }^{3} J_{\mathrm{HH}}=7.0 \mathrm{~Hz}, 1 \mathrm{H}, H 3^{\prime}\right), 7.60-7.57(\mathrm{~m}, 1 \mathrm{H}, \operatorname{Ar} H), 7.53-7.40$ (m, 7H, H3/ArH), 7.36-7.23 (m, 5H, $\operatorname{Ar} H), 7.00\left(\mathrm{~d},{ }^{3} J_{\mathrm{HH}}=8.7\right.$
$\mathrm{Hz}, 1 \mathrm{H}, H 13), 4.16\left(\mathrm{dd},{ }^{3} J_{\mathrm{HP}}=10.2 \mathrm{~Hz},{ }^{4} J_{\mathrm{HH}}=2.8 \mathrm{~Hz}, 1 \mathrm{H}\right.$, allyl $\left.-H \mathrm{t}_{\mathrm{syn}}\right), 2.56\left(\mathrm{~d},{ }^{3} J_{\mathrm{HP}}=14.8 \mathrm{~Hz}, 1 \mathrm{H}\right.$, allyl- $\left.H \mathrm{t}_{\text {anti }}\right), 2.31(\mathrm{~s}$, $1 \mathrm{H}$, allyl- $\left.H \mathrm{c}_{\mathrm{syn}}\right), 0.93\left(\mathrm{~s}, 3 \mathrm{H}\right.$, allyl- $\left.\mathrm{CH}_{3}\right), 0.50(\mathrm{~s}, 1 \mathrm{H}$, allyl$\left.H \mathrm{c}_{\text {anti }}\right) .{ }^{13} \mathrm{C}\left\{{ }^{1} \mathrm{H}\right\}$ NMR $\left(\mathrm{CD}_{2} \mathrm{Cl}_{2}, 126 \mathrm{MHz}\right): \delta(\mathrm{ppm}) 149.1(\mathrm{~d}$, $\left.{ }^{2} J_{\mathrm{CP}}=5.3 \mathrm{~Hz}, C 12^{\prime}\right), 148.4\left(\mathrm{~d},{ }^{2} J_{\mathrm{CP}}=13.1 \mathrm{~Hz}, C 12\right), 145.1(\mathrm{~d}$, $\left.{ }^{2} J_{\mathrm{CP}}=29.7 \mathrm{~Hz}, C 1\right), 135.5,134.5\left(\mathrm{~d},{ }^{1} J_{\mathrm{CP}}=9.4 \mathrm{~Hz}, C 2\right), 134.3$, $\left.133.6\left(\mathrm{~d}, J_{\mathrm{CP}}=4.7 \mathrm{~Hz}\right), 133.5 \mathrm{~d}, J_{\mathrm{CP}}=4.4 \mathrm{~Hz}\right), 133.3,132.6(\mathrm{~d}$, $\left.J_{\mathrm{CP}}=1.4 \mathrm{~Hz}\right), 132.1,131.8\left(\mathrm{~d}, J_{\mathrm{CP}}=0.9 \mathrm{~Hz}\right), 131.6,131.5(\mathrm{~d}$, $\left.{ }^{4} J_{\mathrm{CP}}=1.0 \mathrm{~Hz}, C 2^{\prime}\right), 130.8\left(\mathrm{~d}, J_{\mathrm{CP}}=28.5 \mathrm{~Hz}\right.$, allyl-C), 130.7 $\left(C 14^{\prime}\right), 130.2\left(\mathrm{~d},{ }^{4} J_{\mathrm{CP}}=1.4 \mathrm{~Hz}, C 14\right), 128.6,128.5,128.5$, $128.3,128.1,128.1\left(C 4^{\prime}\right), 127.6\left(\mathrm{~d},{ }^{3} J_{\mathrm{CP}}=4.9 \mathrm{~Hz}, C 4\right), 127.3$, $127.2,127.1,127.0,126.6,126.6,126.5,126.3,126.3,126.2$ $\left(C 3^{\prime}\right), 125.5,125.4,124.5(C 3), 124.1\left(\mathrm{~d},{ }^{3} J_{\mathrm{CP}}=4.2 \mathrm{~Hz}, C 11^{\prime}\right)$, $123.5\left(\mathrm{~d},{ }^{3} J_{\mathrm{CP}}=2.6 \mathrm{~Hz}, C 11\right), 122.5\left(\mathrm{~d},{ }^{3} J_{\mathrm{CP}}=2.3 \mathrm{~Hz}, C 13^{\prime}\right)$, $121.1\left(\mathrm{~d},{ }^{3} J_{\mathrm{CP}}=0.8 \mathrm{~Hz}, C 13\right), 77.8\left(\mathrm{~d},{ }^{2} J_{\mathrm{CP}}=46.5 \mathrm{~Hz}\right.$, allyl-Ct $)$, $57.6\left(\mathrm{~d},{ }^{2} J_{\mathrm{CP}}=5.3 \mathrm{~Hz}\right.$, allyl-Cc), 22.5 (allyl- $\left.\mathrm{CH}_{3}\right) .{ }^{31} \mathrm{P}\left\{{ }^{1} \mathrm{H}\right\}$ NMR $\left(\mathrm{CD}_{2} \mathrm{Cl}_{2}, 202 \mathrm{MHz}\right): \delta(\mathrm{ppm})$ 172.1. HRMS $\left(\mathrm{m} / z, \mathrm{ESI}^{+}\right.$, $\mathrm{MeOH}$ ): found 725.1177 , calcd for $[\mathrm{M}-\mathrm{Cl}]^{+} 725.1176$.

\section{Synthesis of $\left[\operatorname{Pd}(6 a)\left(\eta^{3}-C_{4} H_{7}\right) C l\right](8 a)$}

The same procedure was followed as for 7a. Yield: $26 \mathrm{mg}$ (91\%). MP: $>270{ }^{\circ} \mathrm{C}$. IR (neat): $v$ 3065.2, 1620.2, 1589.0, 1507.6, 1463.0, 1431.3, 1327.7, 1247.8, 1223.0, 1194.2, 1151.8, 1068.9, 1021.8, 943.5, 873.9, 839.0, 807.0, 743.7, 677.2, 637.2, 597.2, 597.8, 560.2 $\mathrm{cm}^{-1} .{ }^{1} \mathrm{H}$ NMR $\left(\mathrm{CD}_{2} \mathrm{Cl}_{2}, 500 \mathrm{MHz}\right): \delta$ $(\mathrm{ppm})$ isomer A (93\%), $7.98\left(\mathrm{~d},{ }^{3} J_{\mathrm{HH}}=9.1 \mathrm{~Hz}, 1 \mathrm{H}, H 4^{\prime}\right), 7.93$ $\left(\mathrm{dd},{ }^{3} J_{\mathrm{HH}}=8.9 \mathrm{~Hz},{ }^{4} J_{\mathrm{HP}}=0.9 \mathrm{~Hz}, 1 \mathrm{H}, H 13\right), 7.91-7.86(\mathrm{~m}, 4 \mathrm{H}$, $\left.H 14 / H 5 / H 15 / H 15^{\prime}\right), 7.78-7.73\left(\mathrm{~m}, 2 \mathrm{H}, H 5^{\prime} / H 4\right), 7.72\left(\mathrm{~d},{ }^{3} J_{\mathrm{HH}}=\right.$ $\left.8.9 \mathrm{~Hz}, 1 \mathrm{H}, H 14^{\prime}\right), 7.63\left(\mathrm{dd},{ }^{3} J_{\mathrm{HH}}=8.6 \mathrm{~Hz},{ }^{3} J_{\mathrm{HP}}=5.4 \mathrm{~Hz}, 1 \mathrm{H}\right.$, $H 3), 7.55\left(\mathrm{ddd},{ }^{3} J_{\mathrm{HH}}=8.1 \mathrm{~Hz},{ }^{3} J_{\mathrm{HH}}=6.8 \mathrm{~Hz},{ }^{4} J_{\mathrm{HH}}=1.1 \mathrm{~Hz}\right.$, $1 \mathrm{H}, \mathrm{Ar} H), 7.50\left(\mathrm{~d},{ }^{3} J_{\mathrm{HH}}=9.1 \mathrm{~Hz}, 1 \mathrm{H}, H 3^{\prime}\right), 7.47-7.24(\mathrm{~m}, 10 \mathrm{H}$, $\left.\operatorname{Ar} H / H 6^{\prime}\right), 7.19\left(\mathrm{~d},{ }^{3} J_{\mathrm{HH}}=8.9 \mathrm{~Hz},{ }^{4} J_{\mathrm{HP}}=1.0 \mathrm{~Hz}, 1 \mathrm{H}, H 13^{\prime}\right)$, $7.14\left(\mathrm{~d},{ }^{3} J_{\mathrm{HH}}=8.4 \mathrm{~Hz}, 1 \mathrm{H}, \mathrm{ArH}\right), 3.97\left(\mathrm{~s}, 3 \mathrm{H}, \mathrm{OCH}_{3}\right), 3.73(\mathrm{dd}$, ${ }^{3} J_{\mathrm{HP}}=10.0 \mathrm{~Hz},{ }^{4} J_{\mathrm{HH}}=3.0 \mathrm{~Hz}, 1 \mathrm{H}$, allyl- $\left.H \mathrm{t}_{\mathrm{syn}}\right), 2.60(\mathrm{~m}, 1 \mathrm{H}$, allyl- $\left.H \mathrm{c}_{\mathrm{syn}}\right), 1.63\left(\mathrm{~d},{ }^{3} J_{\mathrm{HP}}=14.1 \mathrm{~Hz}, 1 \mathrm{H}\right.$, allyl- $\left.H \mathrm{t}_{\text {anti }}\right), 0.92(\mathrm{~s}$, $3 \mathrm{H}$, allyl- $\left.\mathrm{CH}_{3}\right), 0.85\left(\mathrm{~m}, 1 \mathrm{H}\right.$, allyl- $\left.\mathrm{Hc}_{\text {anti }}\right)$; isomer B $(7 \%), 8.05$ $\left(\mathrm{d},{ }^{3} J_{\mathrm{HH}}=9.1 \mathrm{~Hz}, 1 \mathrm{H}, H 4^{\prime}\right), 7.99-7.10(\mathrm{~m}, 20 \mathrm{H}, \operatorname{Ar} H)$, $7.07-7.02(\mathrm{~m}, 1 \mathrm{H}, \operatorname{Ar} H), 6.97\left(\mathrm{~d},{ }^{3} J_{\mathrm{HH}}=8.9 \mathrm{~Hz}, 1 \mathrm{H}, H 13^{\prime}\right)$, $6.74\left(\mathrm{~d},{ }^{3} \mathrm{~J}_{\mathrm{HH}}=8.5 \mathrm{~Hz}, 1 \mathrm{H}, \mathrm{ArH}\right), 4.12\left(\mathrm{~s}, 3 \mathrm{H}, \mathrm{OCH}_{3}\right), 3.87(\mathrm{~m}$, $1 \mathrm{H}$, allyl- $\left.H \mathrm{t}_{\mathrm{syn}}\right), 3.18\left(\mathrm{~m}, 1 \mathrm{H}\right.$, allyl- $\left.H \mathrm{c}_{\mathrm{syn}}\right), 1.94\left(\mathrm{~d},{ }^{3} J_{\mathrm{HP}}=13.0\right.$ $\mathrm{Hz}, 1 \mathrm{H}$, allyl $\left.-H \mathrm{t}_{\text {anti }}\right), 1.70\left(\mathrm{~s}, 3 \mathrm{H}\right.$, allyl- $\left.\mathrm{CH}_{3}\right), 1.35(\mathrm{~m}, 1 \mathrm{H}$, allyl$\left.H \mathrm{c}_{\text {anti) }}\right){ }^{13} \mathrm{C}\left\{{ }^{1} \mathrm{H}\right\}$ NMR $\left(\mathrm{CD}_{2} \mathrm{Cl}_{2}, 126 \mathrm{MHz}\right): \delta(\mathrm{ppm})$ isomer A (93\%), $156.4\left(C 2^{\prime}\right), 149.3\left(\mathrm{~d},{ }^{2} J_{\mathrm{CP}}=5.3 \mathrm{~Hz}, C 12\right), 149.1(\mathrm{~d}$, $\left.{ }^{2} J_{\mathrm{CP}}=12.9 \mathrm{~Hz}, C 12^{\prime}\right), 141.5\left(\mathrm{~d},{ }^{2} J_{\mathrm{CP}}=27.0 \mathrm{~Hz}, C 1\right), 135.5(\mathrm{~d}$, $\left.{ }^{1} J_{\mathrm{CP}}=1.4 \mathrm{~Hz}, C 2\right), 134.2\left(C 9^{\prime}\right), 133.2\left(\mathrm{~d}, J_{\mathrm{CP}}=7.1 \mathrm{~Hz}\right), 133.2$ $\left(\mathrm{d}, J_{\mathrm{CP}}=8.1 \mathrm{~Hz}\right), 132.4\left(\mathrm{~d}, J_{\mathrm{CP}}=1.3 \mathrm{~Hz}\right), 132.1\left(\mathrm{~d}, J_{\mathrm{CP}}=1.4\right.$ $\mathrm{Hz}), 131.8\left(\mathrm{~d}, J_{\mathrm{CP}}=8.1 \mathrm{~Hz}\right), 131.6\left(\mathrm{~d}, J_{\mathrm{CP}}=1.1 \mathrm{~Hz}\right), 131.3(\mathrm{~d}$, ${ }^{2} J_{\mathrm{CP}}=28.8 \mathrm{~Hz}$, allyl-C), $131.0\left(C 4^{\prime}\right), 130.5\left(\mathrm{~d},{ }^{4} J_{\mathrm{CP}}=1.1 \mathrm{~Hz}\right.$, $C 14), 130.1\left(\mathrm{~d},{ }^{4} J_{\mathrm{CP}}=1.3 \mathrm{~Hz}, C 14^{\prime}\right), 128.6,128.5,128.4$, $128.4,128.3,128.2(C 6), 128.0,127.5\left(\mathrm{~d},{ }^{3} J_{\mathrm{CP}}=5.3 \mathrm{~Hz}, C 4\right)$, $127.0\left(C 10^{\prime}\right), 127.0,126.9\left(C 5^{\prime}\right), 126.7,126.4,126.3\left(\mathrm{~d}, J_{\mathrm{CP}}=\right.$ $2.3 \mathrm{~Hz}), 126.2(C 8), 125.4,125.3,125.0\left(\mathrm{~d},{ }^{2} J_{\mathrm{CP}}=2.7 \mathrm{~Hz}, C 3\right)$, $124.5\left(C 6^{\prime}\right), 124.4\left(\mathrm{~d},{ }^{3} J_{\mathrm{CP}}=3.8 \mathrm{~Hz}, C 11\right), 123.7\left(\mathrm{~d},{ }^{3} J_{\mathrm{CP}}=3.0\right.$ $\left.\mathrm{Hz}, C 11^{\prime}\right), 122.9\left(\mathrm{~d},{ }^{3} J_{\mathrm{CP}}=2.4 \mathrm{~Hz}, C 13\right), 121.5\left(C 13^{\prime}\right), 118.8(\mathrm{~d}$, $\left.{ }^{3} J_{\mathrm{CP}}=9.5 \mathrm{~Hz}, C 1^{\prime}\right), 113.1\left(C 3^{\prime}\right), 77.3\left(\mathrm{~d},{ }^{2} J_{\mathrm{CP}}=44.9 \mathrm{~Hz}\right.$, allyl$C \mathrm{t}), 56.2\left(\mathrm{OCH}_{3}\right), 56.1\left(\mathrm{~d},{ }^{2} J_{\mathrm{CP}}=5.3 \mathrm{~Hz}\right.$, allyl-Cc), 22.5 (allyl$\mathrm{CH}_{3}$ ); signals of isomer B could not be observed. ${ }^{31} \mathrm{P}\left\{{ }^{1} \mathrm{H}\right\}$ NMR 
$\left(\mathrm{CD}_{2} \mathrm{Cl}_{2}, 202 \mathrm{MHz}\right): \delta(\mathrm{ppm})$ isomer $\mathrm{A}(93 \%), 173.6$; isomer $\mathrm{B}$ (7\%) 175.6. HRMS ( $\left.\mathrm{m} / \mathrm{z}, \mathrm{ESI}^{+}, \mathrm{MeOH}\right)$ : found 755.1301, calcd for $[\mathrm{M}-\mathrm{Cl}]^{+} 755.1296$.

\section{Synthesis of $\left[\mathrm{Pd}(6 b)\left(\eta^{3}-\mathrm{C}_{4} \mathrm{H}_{7}\right) \mathrm{Cl}\right](8 b)$}

The same procedure was followed as for 7a. Yield: $23 \mathrm{mg}$ (80\%). MP: $>270{ }^{\circ} \mathrm{C}$. IR (neat): $v$ 3066.0, 1619.1, 1587.6, $1506.6,1463.6,1429.5,1323.0,1276.0,1226.1,1199.8,1155.9$, $1117.7,1070.1,1028.0,946.1,867.4,833.3,814.2,751.9$, 706.2, 686.6, 634.8, 606.6, 560.1 $\mathrm{cm}^{-1} .{ }^{1} \mathrm{H} \mathrm{NMR}\left(\mathrm{CD}_{2} \mathrm{Cl}_{2}\right.$, $500 \mathrm{MHz}): \delta(\mathrm{ppm}) 8.02\left(\mathrm{~d},{ }^{3} J_{\mathrm{HH}}=9.1 \mathrm{~Hz}, 1 \mathrm{H}, H 4^{\prime}\right), 8.00-7.97$ (m, 2H,H15/H13'), $7.94\left(\mathrm{~d}^{3}{ }^{3} J_{\mathrm{HH}}=8.9 \mathrm{~Hz}, 1 \mathrm{H}, H 14^{\prime}\right), 7.91(\mathrm{~d}$, $\left.{ }^{3} J_{\mathrm{HH}}=8.1 \mathrm{~Hz}, 1 \mathrm{H}, H 5\right), 7.89\left(\mathrm{~d},{ }^{3} J_{\mathrm{HH}}=8.2 \mathrm{~Hz}, 1 \mathrm{H}, H 15^{\prime}\right), 7.86$ $\left(\mathrm{d},{ }^{3} J_{\mathrm{HH}}=8.7 \mathrm{~Hz}, 1 \mathrm{H}, H 14\right), 7.83\left(\mathrm{~d},{ }^{3} J_{\mathrm{HH}}=8.2 \mathrm{~Hz}, 1 \mathrm{H}, H 5^{\prime}\right)$, $7.65\left(\mathrm{~d},{ }^{3} J_{\mathrm{HH}}=8.7 \mathrm{~Hz}, 1 \mathrm{H}, H 4\right), 7.59\left(\mathrm{ddd},{ }^{3} J_{\mathrm{HH}}=8.1 \mathrm{~Hz},{ }^{3} J_{\mathrm{HH}}\right.$ $\left.=6.6 \mathrm{~Hz},{ }^{4} J_{\mathrm{HH}}=1.0 \mathrm{~Hz}, 1 \mathrm{H}, H 6\right), 7.54-7.51(\mathrm{~m}, 1 \mathrm{H}, H 16)$, $7.49\left(\mathrm{~d},{ }^{3} J_{\mathrm{HH}}=9.1 \mathrm{~Hz}, 1 \mathrm{H}, H 3^{\prime}\right), 7.46-7.39\left(\mathrm{~m}, 3 \mathrm{H}, \mathrm{ArH} / \mathrm{H}_{16} 6^{\prime}\right)$,

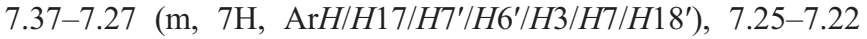
$\left(\mathrm{m}, 1 \mathrm{H}, H 17^{\prime}\right), 7.00\left(\mathrm{~d},{ }^{3} J_{\mathrm{HH}}=8.7 \mathrm{~Hz}, 1 \mathrm{H}, H 13\right), 4.07\left(\mathrm{dd},{ }^{3} J_{\mathrm{HP}}\right.$ $=9.9 \mathrm{~Hz},{ }^{4} J_{\mathrm{HH}}=3.8 \mathrm{~Hz}, 1 \mathrm{H}$, allyl- $\left.H \mathrm{t}_{\mathrm{syn}}\right), 3.87\left(\mathrm{~s}, 3 \mathrm{H}, \mathrm{OCH}_{3}\right)$, $2.35\left(\mathrm{~s}, 1 \mathrm{H}\right.$, allyl- $\left.H \mathrm{c}_{\mathrm{syn}}\right), 2.24\left(\mathrm{~d},{ }^{3} J_{\mathrm{HP}}=13.6 \mathrm{~Hz}, 1 \mathrm{H}\right.$, allyl$\left.H \mathrm{t}_{\text {anti }}\right), 0.84\left(\mathrm{~s}, 3 \mathrm{H}\right.$, allyl- $\left.\mathrm{CH}_{3}\right), 0.32\left(\mathrm{~s}, 1 \mathrm{H}\right.$, allyl- $\left.H \mathrm{c}_{\text {anti }}\right) .{ }^{13} \mathrm{C}$ $\left\{{ }^{1} \mathrm{H}\right\}$ NMR $\left(\mathrm{CD}_{2} \mathrm{Cl}_{2}, 126 \mathrm{MHz}\right): \delta(\mathrm{ppm}) 156.2\left(C 2^{\prime}\right), 149.2(\mathrm{~d}$, $\left.{ }^{2} J_{\mathrm{CP}}=5.3 \mathrm{~Hz}, C 12^{\prime}\right), 148.5\left(\mathrm{~d},{ }^{2} J_{\mathrm{CP}}=12.4 \mathrm{~Hz}, C 12\right), 142.6(\mathrm{~d}$, $\left.{ }^{2} J_{\mathrm{CP}}=29.8 \mathrm{~Hz}, C 1\right), 135.6\left(\mathrm{~d},{ }^{1} J_{\mathrm{CP}}=1.3 \mathrm{~Hz}, C 2\right), 135.5(\mathrm{~d}$, $\left.{ }^{4} J_{\mathrm{CP}}=0.9 \mathrm{~Hz}, C 9^{\prime}\right), 134.0\left(\mathrm{~d}, J_{\mathrm{CP}}=9.2 \mathrm{~Hz}\right), 133.1\left(\mathrm{~d}, J_{\mathrm{CP}}=9.8\right.$ $\mathrm{Hz}), 132.7\left(\mathrm{~d}, J_{\mathrm{CP}}=1.5 \mathrm{~Hz}\right), 132.1\left(\mathrm{~d}, J_{\mathrm{CP}}=1.9 \mathrm{~Hz}\right), 131.8(\mathrm{~d}$, $\left.J_{\mathrm{CP}}=1.3 \mathrm{~Hz}\right), 131.6\left(\mathrm{~d}, J_{\mathrm{CP}}=1.3 \mathrm{~Hz}\right), 131.3\left(\mathrm{~d},{ }^{2} J_{\mathrm{CP}}=26.3 \mathrm{~Hz}\right.$, allyl-C), $130.5\left(\mathrm{~d},{ }^{4} J_{\mathrm{CP}}=0.9 \mathrm{~Hz}, C 14{ }^{\prime}\right), 130.1\left(\mathrm{~d},{ }^{4} J_{\mathrm{CP}}=1.3 \mathrm{~Hz}\right.$, C14), 129.7 (C4'), 128.5 (C15), 128.5 (C15'), 128.5 (C6), 128.3 $(C 5), 128.2\left(C 10^{\prime}\right), 128.0\left(C 5^{\prime}\right), 127.3\left(\mathrm{~d},{ }^{3} J_{\mathrm{CP}}=4.7 \mathrm{~Hz}, C 4\right)$, 127.0, 127.0, 126.9, 126.8, 126.6, 126.6, 126.2 (C17'), 125.5 (C16), $125.3\left(C 16^{\prime}\right), 125.3,125.1\left(\mathrm{~d},{ }^{2} J_{\mathrm{CP}}=1.4 \mathrm{~Hz}, C 3\right), 124.1$ $\left(\mathrm{d},{ }^{3} J_{\mathrm{CP}}=3.8 \mathrm{~Hz}, C 11^{\prime}\right), 123.5,123.4\left(\mathrm{~d},{ }^{3} J_{\mathrm{CP}}=2.8 \mathrm{~Hz}, C 11\right)$, $123.1\left(\mathrm{~d},{ }^{3} J_{\mathrm{CP}}=2.4 \mathrm{~Hz}, C 13^{\prime}\right), 121.4(C 13), 118.2\left(\mathrm{~d},{ }^{3} J_{\mathrm{CP}}=\right.$ $\left.10.5 \mathrm{~Hz}, C 1^{\prime}\right), 113.5\left(C 3^{\prime}\right), 79.3\left(\mathrm{~d},{ }^{2} J_{\mathrm{CP}}=46.1 \mathrm{~Hz}\right.$, allyl-Ct), $59.0\left(\mathrm{~d},{ }^{2} J_{\mathrm{CP}}=4.5 \mathrm{~Hz}\right.$, allyl-Cc), $56.0\left(\mathrm{OCH}_{3}\right), 22.3\left(\right.$ allyl- $\left.\mathrm{CH}_{3}\right)$. ${ }^{31} \mathrm{P}\left\{{ }^{1} \mathrm{H}\right\}$ NMR $\left(\mathrm{CD}_{2} \mathrm{Cl}_{2}, 202 \mathrm{MHz}\right): \delta(\mathrm{ppm})$ 174.4. HRMS $\left(\mathrm{m} / \mathrm{z}, \mathrm{ESI}^{+}, \mathrm{MeOH}\right)$ : found 755.1280 , calcd for $[\mathrm{M}-\mathrm{Cl}]^{+}$ 755.1296 .

\section{Synthesis of $\left[\operatorname{Pd}(6 a)\left(\eta^{3}-C_{4} H_{7}\right)\right] B A r F(9 a)$}

Sodium tetrakis[3,5-bis(trifluoromethyl)phenyl]borate $(33.4 \mathrm{mg}$, $37.7 \mu \mathrm{mol})$ and $\mathbf{8 a}(30.0 \mathrm{mg}, 37.7 \mu \mathrm{mol})$ were dissolved in $\mathrm{CH}_{2} \mathrm{Cl}_{2}(2 \mathrm{~mL})$ and stirred for $30 \mathrm{~min}$. The reaction mixture was filtered through a layer of celite and the solvent removed in vacuo; the intended product was obtained as a yellow solid. Yield: $48.9 \mathrm{mg}(80 \%)$. IR (neat): $v$ 1612.4, 1588.2, 1506.9, $1464.1,1353.8,1273.2,1220.7,1116.5,953.5,882.6,836.8$, $813.3,746.2, \quad 711.9,681.4,638.0,599.2 \mathrm{~cm}^{-1} .{ }^{1} \mathrm{H}$ NMR $\left(\mathrm{CD}_{2} \mathrm{Cl}_{2}, 500 \mathrm{MHz}\right): \delta(\mathrm{ppm})$ isomer $\mathrm{A}, \mathrm{B}, 8.27-7.23(\mathrm{~m}, 2 \mathrm{H}$, $\left.H 4^{\prime \mathrm{B}} / H 4^{\prime \mathrm{A}}\right), \quad 8.14-7.98$ (m, $\left.10 \mathrm{H}, H 5^{\prime} / H 14 / H 15^{\prime} / H 15 / H 14^{\prime}\right)$, 7.93-7.89 (m, 3H, $\left.H 5^{\prime} / H 3^{\prime \mathrm{B}}\right), 7.86-7.80\left(\mathrm{~m}, 3 \mathrm{H}, H 3^{\prime \mathrm{A}} / H 4\right), 7.74$ (br s, 16H, o-BArF), 7.63-7.51 (m, 18H, H6'/H16/H16'/H6/ $\mathrm{ArH} / p-\mathrm{BArF}), 7.48-7.38(\mathrm{~m}, 10 \mathrm{H}, H 13 / \mathrm{ArH}), 7.26-7.19(\mathrm{~m}$, $4 \mathrm{H}, H 7 / \mathrm{Ar} H), 7.13-7.02\left(\mathrm{~m}, 4 \mathrm{H}, H 13^{\prime} / H 3\right), 6.06\left(\mathrm{~d},{ }^{3} J_{\mathrm{HH}}=8.3\right.$
$\mathrm{Hz}, 2 \mathrm{H}, H 8), 4.06\left(\mathrm{~s}, \mathrm{OCH}_{3}{ }^{\mathrm{B}}\right), 4.00\left(\mathrm{~s}, \mathrm{OCH}_{3}{ }^{\mathrm{A}}\right), 3.16\left(\mathrm{br} \mathrm{d},{ }^{3} J_{\mathrm{HP}}\right.$ $=10.7 \mathrm{~Hz}$, allyl- $\left.H \mathrm{t}_{\text {anti }}{ }^{\mathrm{B}}\right), 3.03\left(\mathrm{~s}\right.$, allyl- $\left.H \mathrm{c}_{\mathrm{syn}}{ }^{\mathrm{B}}\right), 2.99$ (s, allyl$H \mathrm{c}_{\text {syn }}{ }^{\mathrm{A}}$ ), $2.90\left(\mathrm{~d},{ }^{3} J_{\mathrm{HP}}=13.2 \mathrm{~Hz}\right.$, allyl- $\left.H \mathrm{t}_{\mathrm{anti}}{ }^{\mathrm{A}}\right), 2.43$ (br d, ${ }^{3} J_{\mathrm{HP}}$ $=8.2 \mathrm{~Hz}$, allyl- $\left.H \mathrm{t}_{\mathrm{syn}} \mathrm{A}^{\mathrm{A}}\right), 2.39\left(\mathrm{~s}\right.$, allyl- $\left.H \mathrm{c}_{\mathrm{anti}} \mathrm{A}^{\mathrm{A}}\right), 2.34$ (br s, allyl$\left.H \mathrm{t}_{\text {syn }}{ }^{\mathrm{B}}\right), 2.22$ (s, allyl- $\left.H \mathrm{c}_{\text {anti }}{ }^{\mathrm{B}}\right), 1.58\left(\mathrm{~s}\right.$, allyl- $\left.\mathrm{CH}_{3}{ }^{\mathrm{A}}\right), 1.30(\mathrm{~s}$, allyl- $\mathrm{CH}_{3}{ }^{\mathrm{B}}$ ); $\mathrm{A}: \mathrm{B}$ ratio from integration of $\mathrm{CH}_{3}$ signals: $52: 48$. ${ }^{11} \mathrm{~B}$ NMR $\left(\mathrm{CD}_{2} \mathrm{Cl}_{2}, 160 \mathrm{MHz}\right): \delta(\mathrm{ppm})-7.6 .{ }^{13} \mathrm{C}\left\{{ }^{1} \mathrm{H}\right\} \mathrm{NMR}$ $\left(\mathrm{CD}_{2} \mathrm{Cl}_{2}, 101 \mathrm{MHz}\right): \delta(\mathrm{ppm})$ isomer $\mathrm{A}, \mathrm{B}, 161.8\left(\mathrm{q},{ }^{1} J_{\mathrm{CB}}=40.8\right.$ $\mathrm{Hz}$, ipso-BArF), $159.0\left(C 2^{\prime \mathrm{B}}\right), 157.2\left(C 2^{\prime \mathrm{A}}\right), 148.1\left(\mathrm{~d},{ }^{3} J_{\mathrm{CP}}=\right.$ $\left.10.1 \mathrm{~Hz}, C 11^{\prime \mathrm{B}}\right), 148.0\left(\mathrm{~d},{ }^{3} J_{\mathrm{CP}}=11.0 \mathrm{~Hz}, C 11^{\prime \mathrm{A}}\right), 147.1(\mathrm{~d}$, $\left.{ }^{3} J_{\mathrm{CP}}=7.7 \mathrm{~Hz}, C 11\right), 142.1\left(\mathrm{~d},{ }^{2} J_{\mathrm{CP}}=41.7 \mathrm{~Hz}, C 1^{\mathrm{B}}\right), 141.9(\mathrm{~d}$, $\left.{ }^{2} J_{\mathrm{CP}}=41.7 \mathrm{~Hz}, C 1^{\mathrm{A}}\right), 137.9\left(\mathrm{~d},{ }^{2} J_{\mathrm{CP}}=9.5 \mathrm{~Hz}\right.$, allyl- $\left.C^{\mathrm{A}}\right), 137.0$ (C2), 134.8 (o-BArF), $134.6\left(C 4^{\prime}\right), 132.8,132.5,132.1,132.0$, $131.7,131.6(C 14), 131.5\left(C 9^{\prime A}\right), 131.4\left(C 9^{\prime B}\right), 131.4\left(C 14^{\prime}\right)$, $130.9,130.8,130.4,130.3(C 4), 130.1\left(C 5^{\prime}\right), 130.0,129.8$, $129.4,128.9$ (qq, $\left.{ }^{2} J_{\mathrm{CF}}=31.2 \mathrm{~Hz},{ }^{4} J_{\mathrm{CF}}=2.8 \mathrm{~Hz}, m-\mathrm{BArF}\right)$, $128.8,128.4\left(C 10^{\prime}\right), 127.5,127.4,127.3,127.1,127.0,126.9$, $126.5,126.4,126.3,126.0,125.0,124.8,124.7\left(\mathrm{q},{ }^{1} J_{\mathrm{CF}}=273.2\right.$ $\left.\mathrm{Hz}, C \mathrm{~F}_{3}\right), 124.1,124.0\left(\mathrm{~d}, J_{\mathrm{CP}}=3.4 \mathrm{~Hz}\right), 123.8,123.7,123.3$, 122.5, 122.3, 120.7 (C3), 120.6 (C13'), 120.3 (C13), 120.1, 117.5 (septet, $\left.{ }^{3} J_{\mathrm{CF}}=4.0 \mathrm{~Hz}, p-\mathrm{BArF}\right), 114.9\left(C 3^{\prime \mathrm{A}}\right), 114.5$ $\left(C 3^{\prime \mathrm{B}}\right), 104.6\left(C 1^{\prime \mathrm{A}}\right), 99.6\left(\mathrm{~d},{ }^{2} J_{\mathrm{CP}}=40.1 \mathrm{~Hz}\right.$, allyl- $\left.C \mathrm{t}^{\mathrm{A}}\right), 57.5$ $\left(\mathrm{OCH}_{3}{ }^{\mathrm{B}}\right), 57.4\left(\mathrm{OCH}_{3}{ }^{\mathrm{A}}\right), 53.2$ (allyl-Cc $\left.{ }^{\mathrm{A}}\right), 22.4$ (allyl- $\mathrm{CH}_{3}{ }^{\mathrm{A}}$ ), 21.6 (allyl- $\mathrm{CH}_{3}{ }^{\mathrm{B}}$ ); not all signals could be observed due to peak broadening and overlap. ${ }^{19} \mathrm{~F}$ NMR $\left(\mathrm{CD}_{2} \mathrm{Cl}_{2}, 471 \mathrm{MHz}\right): \delta$ (ppm) -62.7. ${ }^{31} \mathrm{P}\left\{{ }^{1} \mathrm{H}\right\}$ NMR $\left(\mathrm{CD}_{2} \mathrm{Cl}_{2}, 202 \mathrm{MHz}\right): \delta(\mathrm{ppm})$ isomer $\mathrm{A}$, 177.5; isomer B, 178.0. HRMS $\left(\mathrm{m} / z, \mathrm{ESI}^{+}, \mathrm{MeCN}\right)$ : found 757.1287, calcd for $[\mathrm{M}]^{+} 757.1281$.

\section{Synthesis of $\left[\operatorname{Pd}(6 b)\left(\eta^{3}-C_{4} H_{7}\right)\right] B A r F(9 b)$}

The same procedure was followed as for 9a; the intended product was obtained as a yellow solid. Yield: $54.0 \mathrm{mg}(91 \%)$. IR (neat): $v 1612.1,1588.9,1506.1,1463.5,1353.6,1273.0$, 1219.8, 1116.1, 952.9, 882.4, 836.7, 811.4, 745.5, 711.9, 669.8, 638.6, $602.7 \mathrm{~cm}^{-1}$. ${ }^{1} \mathrm{H} \mathrm{NMR}\left(\mathrm{CD}_{2} \mathrm{Cl}_{2}, 500 \mathrm{MHz}\right): \delta(\mathrm{ppm})$ isomer A,B, $8.22\left(\mathrm{~d},{ }^{3} J_{\mathrm{HH}}=9.2 \mathrm{~Hz}, 1 \mathrm{H}, H 4^{\prime \mathrm{A}}\right), 8.21\left(\mathrm{~d},{ }^{3} J_{\mathrm{HH}}=\right.$ $\left.9.2 \mathrm{~Hz}, 1 \mathrm{H}, H 4^{\prime \mathrm{B}}\right), 8.17-8.01\left(\mathrm{~m}, 10 \mathrm{H}, H 14 / H 14^{\prime} / H 5^{\prime} / \mathrm{Ar} H\right)$, $7.99\left(\mathrm{~d},{ }^{3} J_{\mathrm{HH}}=9.2 \mathrm{~Hz}, 1 \mathrm{H}, H 3^{\mathrm{A}}\right), 7.96-7.89(\mathrm{~m}, 5 \mathrm{H}, \mathrm{ArH} / \mathrm{H} 4 /$ $H 3^{\prime \mathrm{B}}$ ), 7.76 (br s, 16H,o-BArF), 7.64-7.51 (m, 18H, ArH/p$\mathrm{BArF}), 7.49-7.35\left(\mathrm{~m}, 12 \mathrm{H}, \mathrm{Ar} H / H 13^{\mathrm{B}} / H 13^{\prime \mathrm{A}} / H 13^{\mathrm{A}}\right), 7.30-7.22$ $\left(\mathrm{m}, 4 \mathrm{H}, \mathrm{Ar} H / H 7 / H 13^{\prime \mathrm{B}}\right), 7.20\left(\mathrm{dd},{ }^{3} J_{\mathrm{HH}}=8.5 \mathrm{~Hz},{ }^{3} J_{\mathrm{HP}}=6.9\right.$ $\left.\mathrm{Hz}, 1 \mathrm{H}, H 3^{\mathrm{A}}\right), 7.10\left(\mathrm{dd},{ }^{3} J_{\mathrm{HH}}=8.5 \mathrm{~Hz},{ }^{3} J_{\mathrm{HP}}=6.9 \mathrm{~Hz}, 1 \mathrm{H}\right.$, $\left.H 3^{\mathrm{B}}\right), 6.09\left(\mathrm{brd}, 1 \mathrm{H}, H 8^{\mathrm{B}}\right), 6.00\left(\mathrm{~d},{ }^{3} J_{\mathrm{HH}}=8.6 \mathrm{~Hz}, 1 \mathrm{H}, H 8^{\mathrm{A}}\right)$, $3.98\left(\mathrm{~s}, \mathrm{OCH}_{3}{ }^{\mathrm{A}}\right), 3.89\left(\mathrm{~s}, \mathrm{OCH}_{3}{ }^{\mathrm{B}}\right), 3.76\left(\mathrm{~d},{ }^{3} J_{\mathrm{HP}}=13.6 \mathrm{~Hz}\right.$, allyl- $\left.H \mathrm{t}_{\text {anti }}{ }^{\mathrm{A}}\right), 3.00$ (br d, allyl- $\left.H \mathrm{t}_{\mathrm{syn}}{ }^{\mathrm{B}}\right), 2.87$ ( $\mathrm{s}$, allyl- $H \mathrm{c}_{\mathrm{syn}}{ }^{\mathrm{B}}$ ), $2.82\left(\mathrm{~s}\right.$, allyl- $\left.H \mathrm{c}_{\mathrm{syn}}{ }^{\mathrm{A}}\right), 2.63\left(\mathrm{~d},{ }^{3} J_{\mathrm{HP}}=13.5 \mathrm{~Hz}\right.$, allyl $\left.-H \mathrm{t}_{\mathrm{anti}}{ }^{\mathrm{B}}\right)$, 2.32 (s, allyl- $H \mathrm{c}_{\text {anti }} \mathrm{A}$ ), 2.20 (br s, allyl- $H \mathrm{c}_{\text {anti }}{ }^{\mathrm{B}}$ ), 2.19 (d, allyl$\mathrm{Ht}_{\text {syn }}{ }^{\mathrm{A}}$ ), 1.79 (br s, allyl- $\mathrm{CH}_{3}{ }^{\mathrm{B}}$ ), 0.96 (s, allyl- $\left.\mathrm{CH}_{3}{ }^{\mathrm{A}}\right) ; \mathrm{A}: \mathrm{B}$ ratio of $50: 50$ from integration of $\mathrm{OMe}$ resonances (signals of isomer B broadened). ${ }^{11} \mathrm{~B} \mathrm{NMR}\left(\mathrm{CD}_{2} \mathrm{Cl}_{2}, 128 \mathrm{MHz}\right): \delta$ (ppm) -7.6. ${ }^{13} \mathrm{C}\left\{{ }^{1} \mathrm{H}\right\}$ NMR $\left(\mathrm{CD}_{2} \mathrm{Cl}_{2}, 126 \mathrm{MHz}\right): \delta(\mathrm{ppm})$ isomer A,B, 161.8 $\left(\mathrm{q},{ }^{1} J_{\mathrm{CB}}=40.7 \mathrm{~Hz}\right.$, ipso-BArF $), 156.6\left(C 2^{\prime \mathrm{A}}\right), 154.8\left(\mathrm{~d}, J_{\mathrm{CP}}=\right.$ $\left.2.4 \mathrm{~Hz}, C 2^{\prime \mathrm{B}}\right), 148.3\left(\mathrm{~d},{ }^{3} J_{\mathrm{CP}}=13.5 \mathrm{~Hz}, C 11^{\mathrm{A}}\right), 148.3\left(\mathrm{~d},{ }^{3} J_{\mathrm{CP}}=\right.$ $\left.13.3 \mathrm{~Hz}, C 11^{\mathrm{B}}\right), 147.2\left(\mathrm{~d},{ }^{3} J_{\mathrm{CP}}=7.1 \mathrm{~Hz}, C 11^{\prime \mathrm{A}}\right), 147.1\left(\mathrm{~d},{ }^{3} J_{\mathrm{CP}}\right.$ $\left.=7.4 \mathrm{~Hz}, C 11^{\prime \mathrm{B}}\right), 142.4\left(\mathrm{~d},{ }^{2} J_{\mathrm{CP}}=43.5 \mathrm{~Hz}, C 1^{\mathrm{B}}\right), 142.2\left(\mathrm{~d},{ }^{2} J_{\mathrm{CP}}\right.$ $\left.=41.3 \mathrm{~Hz}, C 1^{\mathrm{A}}\right), 138.9\left(\mathrm{~d},{ }^{2} J_{\mathrm{CP}}=10.4 \mathrm{~Hz}\right.$, allyl- $\left.C^{\mathrm{A}}\right), 138.1(\mathrm{~d}$, $J=10.0 \mathrm{~Hz}$, allyl- $\left.C^{\mathrm{B}}\right), 137.1\left(C 2^{\mathrm{B}}\right), 137.0\left(C 2^{\mathrm{A}}\right), 134.9\left(C 4^{\prime \mathrm{A}}\right)$, 
$134.9(o-\mathrm{BArF}), 134.3\left(C 4^{\prime \mathrm{B}}\right), 133.7\left(C 9^{\prime \mathrm{B}}\right), 132.8,132.7\left(C 9^{\prime \mathrm{A}}\right)$ $132.1,131.8,131.7,131.6,131.5,130.7\left(\mathrm{~d},{ }^{3} J_{\mathrm{CP}}=4.7 \mathrm{~Hz}, C 4\right)$, $130.0\left(C 10^{\prime \mathrm{B}}\right), 129.8,129.8,129.7,129.3\left(C 10^{\prime \mathrm{A}}\right), 128.9(\mathrm{qq}$, $\left.{ }^{2} J_{\mathrm{CF}}=31.6 \mathrm{~Hz},{ }^{4} J_{\mathrm{CF}}=2.9 \mathrm{~Hz}, m-\mathrm{BArF}\right), 128.7,128.6,128.0$, $127.6,127.4,127.4,127.0,127.0,126.6,126.5,126.4,125.1$, $124.8,124.7\left(\mathrm{q},{ }^{1} J_{\mathrm{CF}}=272.3 \mathrm{~Hz}, C_{3}\right), 124.3,124.1,123.7$ (d, $\left.{ }^{2} J_{\mathrm{CP}}=1.4 \mathrm{~Hz}, C 3^{\mathrm{B}}\right), 123.7\left(\mathrm{~d},{ }^{2} J_{\mathrm{CP}}=1.4 \mathrm{~Hz}, C 3^{\mathrm{A}}\right), 123.0$, $122.3,122.1,120.7\left(\mathrm{~d},{ }^{3} J_{\mathrm{CP}}=1.0 \mathrm{~Hz}, C 13^{\mathrm{B}}\right), 120.6\left(\mathrm{~d},{ }^{3} J_{\mathrm{CP}}=\right.$ $\left.1.0 \mathrm{~Hz}, C 13^{\mathrm{A}}\right), 120.1\left(\mathrm{~d},{ }^{3} J_{\mathrm{CP}}=2.1 \mathrm{~Hz}, C 13^{\prime \mathrm{A}}\right), 119.8\left(C 13^{\prime \mathrm{B}}\right)$, 117.5 (septet, ${ }^{3} J_{\mathrm{CF}}=4.0 \mathrm{~Hz}, p$-BArF), $115.9\left(C 3^{\mathrm{B}}\right), 115.3$ $\left(C 3^{\prime \mathrm{A}}\right), 104.5\left(C 1^{\prime \mathrm{B}}\right), 103.4\left(C 1^{\prime \mathrm{A}}\right), 99.5\left(\mathrm{~d},{ }^{2} J_{\mathrm{CP}}=41.1 \mathrm{~Hz}\right.$, allyl- $\left.C \mathrm{t}^{\mathrm{A}}\right), 96.9\left(\mathrm{br}\right.$, allyl- $\left.C \mathrm{t}^{\mathrm{B}}\right), 57.6\left(\mathrm{OCH}_{3}{ }^{\mathrm{A}}\right), 57.3\left(\mathrm{OCH}_{3}{ }^{\mathrm{B}}\right)$, 56.4 (allyl- $C \mathrm{c}^{\mathrm{B}}$ ), 54.9 (allyl- $C \mathrm{c}^{\mathrm{A}}$ ), 22.6 (allyl- $\mathrm{CH}_{3}{ }^{\mathrm{B}}$ ), 21.5 (allyl$\mathrm{CH}_{3}{ }^{\mathrm{A}}$ ); not all signals could be observed due to peak broadening and overlap. ${ }^{19} \mathrm{~F}$ NMR $\left(\mathrm{CD}_{2} \mathrm{Cl}_{2}, 471 \mathrm{MHz}\right): \delta(\mathrm{ppm})-62.7 .{ }^{31} \mathrm{P}$ $\left\{{ }^{1} \mathrm{H}\right\} \mathrm{NMR}\left(\mathrm{CD}_{2} \mathrm{Cl}_{2}, 202 \mathrm{MHz}\right): \delta(\mathrm{ppm})$ isomer $\mathrm{A}, 178.9$; isomer $\mathrm{B}, 179.1$. HRMS $\left(m / z, \mathrm{ESI}^{+}, \mathrm{MeCN}\right)$ : found 757.1296, calcd for $[\mathrm{M}]^{+} 757.1281$.

\section{Asymmetric palladium-catalyzed hydrosilylation of styrene}

$\left[\operatorname{Pd}\left(\eta^{3}-\mathrm{C}_{3} \mathrm{H}_{4}\right) \mathrm{Cl}\right]_{2}(4.6 \mathrm{mg}, 0.0125 \mathrm{mmol}, 0.125 \mathrm{~mol} \%)$, ligand $(0.25 \mathrm{~mol} \%)$ and styrene $(1.2 \mathrm{~mL}, 1.0 \mathrm{~g}, 10.0 \mathrm{mmol})$ were stirred at room temperature for $20 \mathrm{~min}$. Trichlorosilane $(1.2 \mathrm{~mL}$, $1.6 \mathrm{~g}, 12.0 \mathrm{mmol}$ ) was added and the reaction was stirred at room temperature for the appropriate time. The conversion of the reaction was followed by ${ }^{1} \mathrm{H}$ NMR spectroscopy. The product was purified by Kugelrohr distillation (reduced pressure, $\left.150{ }^{\circ} \mathrm{C}\right)$.

Trichloro(1-phenylethyl)silane (400 mg, $1.67 \mathrm{mmol})$ was dissolved in $\mathrm{MeOH}(30 \mathrm{~mL})$ and THF $(30 \mathrm{~mL}) . \mathrm{K}_{2} \mathrm{CO}_{3}(1.40 \mathrm{~g}$, $10.1 \mathrm{mmol}), \mathrm{KF}$ (600 mg, $10.3 \mathrm{mmol})$ and $35 \% \mathrm{H}_{2} \mathrm{O}_{2}(1.8 \mathrm{~mL})$ were added subsequently and left to stir overnight. The solution was filtered, water was added and the product was extracted with diethyl ether three times. The combined organic washings were dried over $\mathrm{MgSO}_{4}$ and the crude product was purified by column chromatography on silica (hexane/ethyl acetate, $4: 1, R_{\mathrm{f}}=0.20$ ). The enantiomeric excess was measured by chiral HPLC (Column Daicel Chiralcel OD; flow rate: $0.5 \mathrm{~mL} \mathrm{~min}^{-1}$; hexane/ 2-propanol, $95: 5$; retention times: $(R) t_{1}=19.3 \mathrm{~min},(S) t_{2}=$ $22.3 \mathrm{~min})$. The absolute configuration was assigned by comparing the retention times to literature data. ${ }^{23}$

\section{Acknowledgements}

We thank the EPSRC for a Career Acceleration Fellowship (L.J.H.), Studentship (A.F.), an Equipment Grant (R.W.H) and its National Mass Spectrometry Service Centre, Swansea, UK, and IRCSET for a Scholarship (R.M.H.).

\section{Notes and references}

1 Selected review articles: (a) F. Lagasse and H. B. Kagan, Chem. Pharm. Bull., 2000, 48, 315; (b) I. V. Komarov and A. Börner, Angew. Chem. Int. Ed., 2001, 40, 1197; (c) T. Jerphagnon, J.-L. Renaud and C. Bruneau, Tetrahedron: Asymmetry, 2004, 15, 2101; (d) G. Erre, S. Enthaler, K. Junge, S. Gladiali and M. Beller, Coord. Chem. Rev., 2008, 252, 471; (f) M. T. Reetz, Angew. Chem., Int. Ed., 2008, 47, 2556; (f) J. Ansell and M. Wills, Chem. Soc. Rev., 2002, 31, 259.

2 (a) J. W. Han and T. Hayashi, Tetrahedron: Asymmetry, 2010, 21, 2193; (b) T. Hayashi, Acc. Chem. Res., 2000, 33, 354.

3 (a) J. F. Teichert and B. L. Feringa, Angew. Chem., Int. Ed., 2010, 49, 2486; (b) B. L. Feringa, Acc. Chem. Res., 2000, 33, 346.

4 Y. (A.) Xu, G. C. Clarkson, G. Docherty, C. L. North, G. Woodward and M. Wills, J. Org. Chem., 2005, 70, 8079.

5 K. V. Katti, N. Pillarsetty and K. Raghuraman, Top. Curr. Chem., 2003, 229, 121.

6 See for example: (a) M. Brynda, Coord. Chem. Rev., 2005, 249, 2013; (b) N. Pillarsetty, K. Raghuraman, C. L. Barnes and K. V. Katti, J. Am. Chem. Soc., 2005, 127, 331; (c) W. Henderson and S. R. Alley, J. Organomet. Chem., 2002, 656, 120; (d) M. Brynda, M. Geoffroy and G. Bernardinelli, Chem. Commun., 1999, 961.

7 R. M. Hiney, L. J. Higham, H. Müller-Bunz and D. G. Gilheany, Angew. Chem., Int. Ed., 2006, 45, 7248.

8 B. Stewart, A. Harriman and L. J. Higham, Organometallics, 2011, 30, 5338.

9 A. Ficks, I. Martinez-Botella, B. Stewart, R. W. Harrington, W. Clegg and L. J. Higham, Chem. Commun., 2011, 47, 8274.

10 N. Z. Weferling, Z. Anorg. Allg. Chem., 1987, 548, 55.

11 Note that $N$-chlorosuccinimide, phosgene, triphosgene and chlorine were not suitable reagents for the reaction, giving mixtures of compounds: (a) H. Kischkel and G.-V. Röschenthaler, Chem. Ber., 1985, 118, 4842; (b) W. A. Henderson Jr., S. A. Buckler, N. E. Day and M. Grayson, J. Org. Chem., 1961, 26, 4770; (c) L. Dahlenburg and A. Kaunert, Eur. J. Inorg. Chem., 1998, 885.

12 The presence of trace quantities of $\mathrm{HCl}$ and $\mathrm{H}_{2} \mathrm{O}$ (in unstabilised bench $d$-chloroform for instance) did lead to H-phosphinate formation.

13 (a) T. Hayashi, H. Iwamura, M. Naito, Y. Matsumoto, Y. Uozumi, M. Miki and K. Yanagi, J. Am. Chem. Soc., 1994, 116, 775; (b) P. G. A. Kumar, P. Dotta, R. Hermatschweiler, P. S. Pregosin, A. Albinati and S. Rizzato, Organometallics, 2005, 24, 1306.

14 M. C. Bonnet, S. Agbossou and I. Tkatchenko, Acta Cryst, 1987, C43, 445.

15 A. Grabulosa, G. Muller, J. I. Ordinas, A. Mezzetti, M. A. Maestro, M. Font-Bardia and X. Solans, Organometallics, 2005, 24, 4961.

16 S. Filipuzzi, P. S. Pregosin, M. J. Calhorda and P. J. Costa, Organometallics, 2008, 27, 2949 and references therein.

17 (a) C. Breutel, P. S. Pregosin, R. Salzmann and A. Togni, J. Am. Chem. Soc., 1994, 116, 4067; (b) B. E. Ketz, A. P. Cole and R. M. Waymouth, Organometallics, 2004, 23, 2835; (c) H. M. Peng, G. Song, Y. Li and X. Li, Inorg. Chem., 2008, 47, 8031 .

18 (a) A. Guerrero, F. A. Jalon, B. R. Manzano, A. Rodriguez, R. M. Claramunt, P. Cornago, V. Milata and J. Elguero, Eur. J. Inorg. Chem., 2004, 549; (b) A. Gogoll, H. Grennberg and A. Axén, Organometallics, 1997, 16, 1167; (c) F. A. Jalón, B. R. Manzano and B. MorenoLara, Eur. J. Inorg. Chem., 2005, 100; (d) V. Montoya, J. Pons, J. GarciaAntón, X. Solans, M. Font-Bardía and J. Ros, Organometallics, 2007, 26, 3183; (e) A. Ficks, C. Sibbald, M. John, S. Dechert and F. Meyer, Organometallics, 2010, 29, 1117.

19 (a) P. Kočovský, Š. Vyskočil, I. Císařová, J. Sejbal, I. Tišlerová, M. Smrčina, G. C. Lloyd-Jones, S. C. Stephen, C. P. Butts, M. Murray and V. Langer, J. Am. Chem. Soc., 1999, 121, 7714; (b) P. Dotta, P. G. A. Kumar, P. S. Pregosin, A. Albinati and S. Rizzato, Organometallics, 2004, 23, 4247; (c) P. Dotta, P. G. A. Kumar, P. S. Pregosin, A. Albinati and S. Rizzato, Helv. Chim. Acta, 2004, 87, 272; (d) P. Dotta, P. G. A. Kumar, P. S. Pregosin, A. Albinati and S. Rizzato, Organometallics, 2003, 22, 5345; (e) I. S. Mikhel, H. Rüegger, P. Butti, F. Camponovo, D. Huber and A. Mezzetti, Organometallics, 2008, 27, 2937.

20 (a) P. S. Pregosin, Chem. Commun., 2008, 4875; (b) P. S. Pregosin, Coord. Chem. Rev., 2008, 252, 2156.

21 (a) S. E. Gibson and M. Rudd, Adv. Synth. Catal., 2007, 349, 781; (b) L. Tietze, H. Ila and H. P. Bell, Chem. Rev., 2004, 104, 3453.

22 P. Dotta, P. G. A. Kumar, P. S. Pregosin, A. Albinati and S. Rizzato, Organometallics, 2004, 23, 2295.

23 M. Ito, Y. Endo, N. Tejima and T. Ikariya, Organometallics, 2010, 29, 2397.

24 J. C. Cobas and M. Martín-Pastor, EXSYCalc 1.0; Mestrelab Research, Escondido, CA, 2007. 